\title{
Fast Parametric Elastic Image Registration
}

\author{
Jan Kybic, Member, IEEE, and Michael Unser, Fellow, IEEE
}

\begin{abstract}
We present an algorithm for fast elastic multidimensional intensity-based image registration with a parametric model of the deformation. It is fully automatic in its default mode of operation. In the case of hard real-world problems, it is capable of accepting expert hints in the form of soft landmark constraints. Much fewer landmarks are needed and the results are far superior compared to pure landmark registration. Particular attention has been paid to the factors influencing the speed of this algorithm. The B-spline deformation model is shown to be computationally more efficient than other alternatives.

The algorithm has been successfully used for several two-dimensional (2-D) and three-dimensional (3-D) registration tasks in the medical domain, involving MRI, SPECT, CT, and ultrasound image modalities. We also present experiments in a controlled environment, permitting an exact evaluation of the registration accuracy. Test deformations are generated automatically using a random hierarchical fractional wavelet-based generator.
\end{abstract}

Index Terms-Elastic registration, image registration, landmarks, splines.

\section{INTRODUCTION}

I MAGE registration is the task of finding a correspondence function mapping coordinates from a reference image to coordinates of homologous points in a test image [1]. We call the registration elastic [2] if the family of correspondence functions is sufficiently general, capable of expressing essentially arbitrary nonlinear relations. ${ }^{1}$ Image registration is applied in the areas of motion analysis [4]-[6], video compression and coding [7], object tracking [8], or image stabilization. It leads to algorithms for segmentation [9], depth reconstruction from stereo images [10], [11], and for general 3-D reconstruction. In the biomedical domain, there is a frequent need for comparing images for analysis and diagnostic purposes. This is accomplished by registering the images and aligning them by warping using the correspondence function identified. Applications include intra-subject [12], inter-subject [13], [14], and inter-modality analysis [15], [16], [17], registration with annotated atlases [18], [19], quantification and qualification of feature shapes and sizes [20], distortion compensation [21], [22] and motion detection [23], [24] and compensation [25].

Manuscript received September 6, 2001; revised February 12, 2003. The associate editor coordinating the review of this manuscript and approving it for publication was Dr. Patrick Perez.

J. Kybic was with the Biomedical Imaging Group, LIB, Swiss Federal Institute of Technology Lausanne, CH-1015 Lausanne EPFL, Switzerland. He is now with the Center for Machine Perception, Department of Cybernetics, Faculty of Electrical Engineering, Czech Technical University, Prague 6, Czech Republic (e-mail: kybic@ieee.org).

M. Unser was with the Biomedical Imaging Group, LIB, Swiss Federal Institute of Technology Lausanne, CH-1015 Lausanne EPFL, Switzerland.

Digital Object Identifier 10.1109/TIP.2003.813139

${ }^{1}$ Note that elasticity is used here in a wider sense than just the mechanical linear elasticity [3].
Various nonlinear registration algorithms for brain warping applications are presented by Warfield et al. [9]. Bayesian interpretation of elastic matching was reviewed by Gee [19], also in the context of human neuroanatomy. Articles by Van den Elsen et al. [26] and Maintz and Viergever [27] contain a very comprehensive and detailed classification of available methods for medical imaging applications. Lester and Arridge [28] treat the hierarchical aspects of the algorithms.

The deformation models of elastic registration algorithms fall into two basic categories. The first type are nonparametric, local methods-the deformation function is basically unconstrained and belongs to a very large and unrestrictive functional space. These methods can be formulated as variational, defining a scalar criterion that completely determines the final solution [2]. More generally, they can be also expressed using partial differential equations (PDE) [29]-[32].

The presented algorithm belongs to a second group of methods that use parametric models, representing the deformation by a moderate number of parameters, often in the multiscale setting. Specific examples include hierarchical basis functions by Moulin et al. [7], quadtree-splines [5], multiresolution subspaces [33], [34], and wavelets [35], [36]. Splines are well suited for this kind of problems; they have appeared in various incarnations. In this paper we use a multiresolution B-spline representation, as was initially suggested in the pioneering work of Szeliski et al. [10], [5].

\section{A. Proposed Algorithm}

The algorithm described in this article is a synthesis of several ideas. First, it is a generalization to multiple dimensions of the unidirectional registration algorithm we described in [22]. Its main features are the use of B-splines to describe both the image and the deformation, a double multiresolution strategy (for both the image and the deformation), a scalar pixel-based difference measure, and an iterative multidimensional optimization algorithm [37], [38]. The deformation model has been generalized and the whole algorithm re-engineered for faster execution.

Second, we present the idea of semi-automatic registration, targeted to more difficult registration problems. We ask an expert to identify a small number of corresponding points in both images. The points are also called landmarks [3], [12], [39], [40]. We add a term to the data part of the criterion, to steer the algorithm toward the correct solution indicated by the landmarks.

\section{B. Organization of This Article}

In Section II, we describe the concept of registration by minimization, the difference measure, the B-spline image model, 
and the structure of the deformation model. In Section III we justify our choice of B-splines as basis functions for the deformation model. We present the optimization method in Section IV, where we also describe the multiresolution strategy. Section V is devoted to the semi-automatic mode incorporating landmark information into the global criterion. We deal with implementation issues in Section VI and present experiments and applications in Section VII. For more details, we refer the reader to the first author's thesis report [38] and its associated web page.

\section{PROBLEM Formulation}

The input images are given as two $N$-dimensional discrete signals $f_{r}(\mathbf{i})$ and $f_{t}(\mathbf{i})$, where $\mathbf{i} \in I \subset \mathbb{Z}^{N}$, and $I$ is an $N$ dimensional discrete interval representing the set of all pixel coordinates in the image. We call $f_{r}$ and $f_{t}$ reference and test images, respectively. We suppose that the test image is a geometrically deformed version of the reference image, and vice versa. ${ }^{2}$ This is to say that the points with the same coordinate $\mathbf{x}$ in the reference image $f_{r}(\mathbf{x})$ and in the warped test image $f_{w}(\mathbf{x})=f_{t}^{c}(\mathbf{g}(\mathbf{x}))$ should correspond. Here, $f_{t}^{c}$ is a continuous version of the test image and $\mathbf{g}(\mathbf{x})$ is a deformation (correspondence) function to be identified

\section{A. Cost Function}

The two images $f_{r}, f_{w}$ will not be identical because of noise and also because the assumption that there is a geometrical mapping between the two images is not necessarily correct. Therefore, we define the solution to our registration problem as the result of the minimization $\mathbf{g}=\arg \min _{\mathbf{g} \in G} E(\mathbf{g})$, where $G$ is the space of all admissible deformation functions $\mathrm{g}$. We have chosen the SSD (sum of squared differences) criterion

$$
\begin{aligned}
E & =\frac{1}{\|I\|} \sum_{\mathbf{i} \in I} e_{\mathbf{i}}^{2}=\frac{1}{\|I\|} \sum_{\mathbf{i} \in I}\left(f_{w}(\mathbf{i})-f_{r}(\mathbf{i})\right)^{2} \\
& =\frac{1}{\|I\|} \sum_{\mathbf{i} \in I}\left(f_{t}^{c}(\mathbf{g}(\mathbf{i}))-f_{r}(\mathbf{i})\right)^{2}
\end{aligned}
$$

because it is fast to evaluate and yields a smooth criterion surface which lends itself well to optimization. Minimization of (1) yields the optimal solution $g$ in the ML (maximum likelihood) sense under the assumption that $f_{r}$ is a deformed (warped) version of $f_{t}$ with i.i.d. (independent and identically distributed) Gaussian noise added to each pixel. The SSD criterion proved to be robust enough, especially if preprocessing was used to equalize the image values-we mostly applied high-pass filtering and histogram normalization [22]. In principle, there is no difficulty in extending our method for more sophisticated pixelbased similarity measures, such as information-based measures [41], especially mutual information [17], or weighted $\ell_{p}$ norms. Only the evaluation of the criterion and its derivatives (gradient) needs to be changed.

\footnotetext{
${ }^{2}$ In the multimodal case, which we are not considering here, there can be also an intensity mapping between the two images.
}

\section{B. Image Interpolation}

In accordance with [22], we choose to interpolate the image using uniform B-splines: ${ }^{3}$

$$
f_{t}^{c}(\mathbf{x})=\sum_{\mathbf{i} \in I_{b} \subset \mathbb{Z}^{N}} b_{\mathbf{i}} \beta_{n}(\mathbf{x}-\mathbf{i})
$$

where $\beta_{n}$ is a tensor product of $\mathrm{B}$-splines of degree $n$, that is $\beta_{n}(\mathbf{x})=\prod_{k=1}^{N} \beta_{n}\left(x_{k}\right)$, with $\mathbf{x}=\left(x_{1}, \ldots, x_{N}\right)$.

\section{Deformation Model Structure}

So far, we have considered the deformation function $\mathrm{g}$ to be an arbitrary admissible function $\mathbb{R}^{N} \rightarrow \mathbb{R}^{N}$. We will restrict it now to a family of functions described by a finite number of parameters $\mathbf{c}_{\mathbf{j}}$ :

$$
\mathrm{g}(\mathrm{x})=\mathbf{x}+\sum_{\mathbf{j} \in J} \mathbf{c}_{\mathbf{j}} \varphi_{\mathbf{j}}(\mathbf{x})
$$

where $J$ is a set of parameter indexes and $\varphi_{\mathbf{j}}$ are the corresponding basis functions. This transforms a variational problem into a much easier finite-dimensional minimization problem, for which numerous algorithms exist [43]. Moreover, the restriction of the family $G$ of all possible functions g can already guarantee some useful properties, such as the regularity (smoothness) of the solution. Note that the addition of $\mathbf{x}$ in the above equation makes the set of zero parameters correspond to identity.

\section{Existence, Unicity, and Regularization}

Note that the criterion $E$ is nonnegative and continuous and $f_{t}^{c}$ is periodic due to boundary conditions. Consequently, $E$ has a minimum; i.e., the proposed problem has a solution. However, depending on the images at hand, the solution does not have to be unique and there can be local minima. Fortunately, this does not pose problems in practice thanks to a multiresolution approach (Section IV-B) which smoothes out images at coarse levels and brings us sufficiently close to the solution at fine resolution levels. The algorithm will find a solution if started within the attraction basin of that solution. The virtual springs (Section V) play a role of an a priori information and a regularization term; extra regularization can be applied [44] if desired.

\section{DEFORMATION BASIS}

The purpose of this section is to motivate our choice of (cubic) B-splines [42] as the most adequate basis functions $\varphi_{\mathbf{j}}$ to represent the deformation in model (3). The alternative possibilities that come to mind are polynomials [45], harmonic functions [18], [46], radial basis functions [3], [47], and wavelets [35], [48], [49].

It is highly desirable to have as few basis functions as possible to contribute to each particular point, while keeping the

\footnotetext{
${ }^{3}$ Uniform symmetric B-splines [42] of degree $n$ are piecewise polynomials of degree $n$. The polynomial pieces are delimited by uniformly placed knots. B-splines of degree $n$ have continuous derivatives up to order $n-1$ everywhere. Their integer shifts form a basis. The first (degree zero) symmetric B-spline is defined as $\beta_{0}(x)=1$ for $x \in(-1 / 2,1 / 2)$ and 0 otherwise. Higher order $\mathrm{B}$-splines are defined by recursively as $\beta_{n+1}=\beta_{n} * \beta_{0}$; and their support is $(-(n+1) / 2,+(n+1) / 2)$.
} 


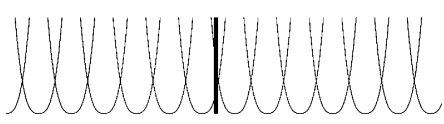

(a)

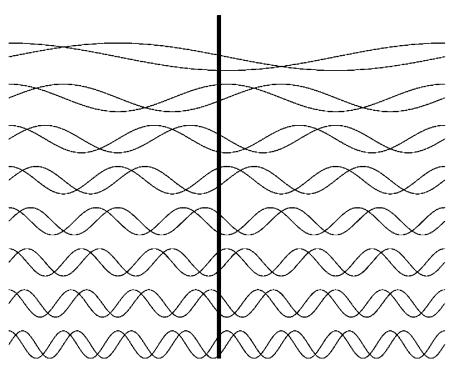

(b)

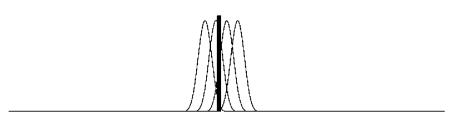

(c)

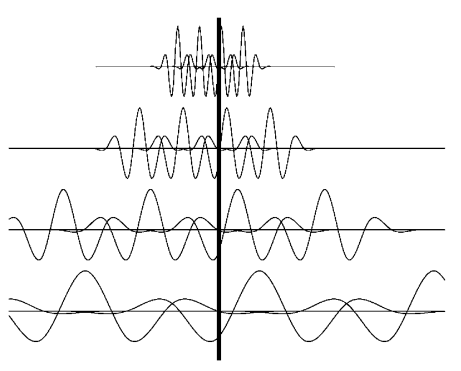

(d)

Fig. 1. Basis functions involved in evaluating the value of a 1-D function at one point (denoted by a vertical line): (a) radial basis functions $|x|^{3}$, (b) harmonic functions, (c) cubic B-splines, and (d) cubic B-spline wavelets.

approximation quality. First, short basis functions have small overlap. This reduces the interdependency between the coefficients (parameters) and consequently makes the minimization problem easier to solve. Small overlap also makes the Hessian (the matrix of second partial derivatives, needed for some optimizers) more sparse and therefore potentially faster to invert.

Second, the size of the support of the basis functions directly influences the speed of the calculation. The evaluation of the deformation function (3) at $N_{\text {pix }}$ points costs $O\left(N_{\text {pix }} N_{\text {cnt }}\right)$ operations, where $N_{\mathrm{cnt}}$ is the number of functions $\varphi_{\mathbf{j}}$ contributing to a single point. ${ }^{4}$ The cost of evaluating the gradient $\nabla_{\mathbf{c}} E$ of the criterion $E$ with respect to the coefficients is higher but asymptotically equivalent, because each of the $N_{\text {pix }}$ pixels contributes to exactly $N_{\text {cnt }}$ components of the gradient. Note that this cost is independent of the total number $N_{\text {tot }}$ of the basis functions (unless $N_{\text {tot }}=N_{\text {cnt }}$ ). The cost of evaluating the Hessian is $O\left(N_{\text {pix }} N_{\text {cnt }}^{2}\right)$ operations. (See also Section VI-A.)

Fig. 1 shows the generating functions needed to calculate a value at one point (denoted by the vertical bar) for various bases; only functions that are nonzero at that point are considered. Except for the Fourier basis, we choose basis functions of the

\footnotetext{
${ }^{4} \mathrm{We}$ assume that the cost of evaluating the basis function itself is constant or that their values can be precalculated.
}

same degree (cubic), generating the same space. We see clearly that the least number of contributing functions (four) is in the B-spline case. This effect turns out to be even more dramatic in higher dimensions.

The reasoning above rules out the polynomials because no fast algorithm is known for their evaluation and the brute-force evaluation is slow due to their long support. As for the radial basis functions, although there are algorithms with reduced asymptotical complexity for evaluation of radial basis functions [50]-[53], their overhead is still nonnegligible. We decided against the harmonic (Fourier) basis functions because of their lack of localization (the fact that any two of them overlap). Another argument against the Fourier basis is that it cannot express linear functions (affine deformations). The only two remaining candidate basis are therefore B-splines and B-spline wavelets.

\section{A. Splines Versus Wavelets}

To make a fair comparison between B-spline and wavelet bases, we consider compactly supported cubic B-spline wavelets [54] spanning the same cubic spline space. First, let us analyze the task of evaluating the deformation at a single point. For simplicity, we will work in 1-D. There are only four participating B-splines altogether while there are four participating B-spline wavelets at each level, plus four scaling functions (cubic B-splines) at the coarsest level. Second, to evaluate the deformation at a set of equally spaced points (this corresponds to a regular grid in multiple dimensions), the direct B-spline representation is also the most efficient, the interpolation requiring only four multiplications per pixel. This is better than all alternatives available when using the B-spline wavelets, including iterative filterbank and FFT-based algorithms.

Note that the complexity of evaluation of the gradient of the criterion corresponds to the complexity of the evaluation of the deformation because the same type of formula is involved (see Section VI-A).

\section{B. B-Spline Deformation Model}

The B-spline deformation model is obtained by substituting a scaled version of the B-spline (or tensor product thereof) in (3)

$$
\mathbf{g}(\mathbf{x})=\mathbf{x}+\sum_{\mathbf{j} \in I_{c} \subset \mathbb{Z}^{N}} \mathbf{c}_{\mathbf{j}} \beta_{n_{m}}(\mathbf{x} / \mathbf{h}-\mathbf{j})
$$

where $n_{m}$ is the degree of splines used, $\mathbf{h}$ is the knot spacing, and the division is taken elementwise. This corresponds to placing the knots on a regular grid over the image. We require the node spacing $\mathbf{h}$ to be integer, which together with the separability of $\beta_{n_{m}}(\mathbf{x})$ implies that the values of the B-spline $\beta_{n_{m}}(x)$ are only needed at a very small number of points $\left(n_{m}+1\right) \mathbf{h}$ and can be precalculated. We can evaluate $\mathbf{g}$ on the whole grid with the cost of only $N\left(n_{m}+1\right)$ multiplications per pixel.

The B-spline model has good approximation properties and is fast to evaluate. It is physically plausible, for example cubic splines minimize the 'strain energy' $\left\|g^{\prime \prime}\right\|_{2}$ [55], [56]. It can encode all affine transformations, including rigid body motion. 
Moreover, B-splines are scalable in the sense that any coarse level deformation can be represented at a finer scale without any loss of information given an integer ratio between scales. The expansion operator (Section VI-C) is therefore exact.

\section{OPTIMIZATION STRATEGY}

\section{A. Optimization Algorithm}

Recall from (1) and (4) that we need to minimize a criterion $E$ with respect to a finite number of parameters $\mathbf{c}$. To determine which of the many available algorithms performs best in our context, we tested four local iterative algorithms which can be cast into a common framework: At each step $i$ we take the actual estimate $\mathbf{c}^{(i)}$ and calculate a proposed update $\Delta \mathbf{c}^{(i)}$. If the step is successful, then the proposed point is accepted, $\mathbf{c}^{(i+1)}=\mathbf{c}^{(i)}+\Delta \mathbf{c}^{(i)}$. Otherwise, a more conservative update $\Delta \mathbf{c}^{(i)}$ is calculated, and the test is repeated.

1) Gradient descent with feedback step size adjustment with update rule: $\Delta \mathbf{c}^{(i)}=-\mu \nabla_{\mathbf{c}} E\left(\mathbf{c}^{(i)}\right)$. After a successful step, $\mu$ is multiplied by $\mu_{f}$, otherwise it is divided by $\mu_{f}^{\prime} .{ }^{5}$

2) Gradient descent with quadratic step size estimation. We choose a step size $\mu^{*}$ minimizing the following approximation of the criterion around $\mathbf{c}^{(i)}: E\left(\mathbf{c}^{(i)}+\mathbf{x}\right)=E\left(\mathbf{c}^{(i)}\right)$ $+\mathbf{x}^{T} \nabla_{\mathbf{c}} E\left(\mathbf{c}^{(i)}\right)+\alpha\|\mathbf{x}\|^{2}$, where $\alpha$ is identified from the two last calculated criterion values $E$. As a fallback strategy, the previous step size is divided by $\mu_{f}^{\prime}$, as above.

3) Conjugated gradient. This algorithm [43] chooses its descent directions to be mutually conjugate so that moving along one does not spoil the result of previous optimizations. To work well, the step size $\mu$ has to be chosen optimally. Therefore, at each step, we need to run another internal one-dimensional minimization routine which finds the optimal $\mu$; this makes it the slowest algorithm in our setting.

4) Marquardt-Levenberg. The most effective algorithm in the sense of the number of iterations was a regularized Newton method inspired by the Marquardt-Levenberg algorithm (ML), as in [22]. Various approximations of the Hessian matrix $\nabla_{\mathbf{c}}^{2} E$ were examined (see also Section VI-A).

As the behavior of all optimizers is comparable at the beginning of the optimization process (see Fig. 2), the main factor determining the speed is the cost of a single iteration. The evaluation costs are presented in Table I; for the ML algorithm, the cost of the Hessian matrix inversion (which grows with the cube of the number of parameters) must be added. It follows that the gradient descent (GD) iterations are the least costly, the difference between the two variant being minimal. We therefore recommend to use the GD algorithm with the quadratic step size estimation (which works better than the feedback adjustment) and we use it for experiments in the remainder of the paper. One additional pleasant property of the GD algorithm is its tendency to leave uninfluential coefficients intact, unlike the ML algorithm. Consequently, less regularization is needed for the GD algorithm.

\footnotetext{
${ }^{5}$ We used $\mu_{f}=10$ and $\mu_{f}^{\prime}=15$.
}

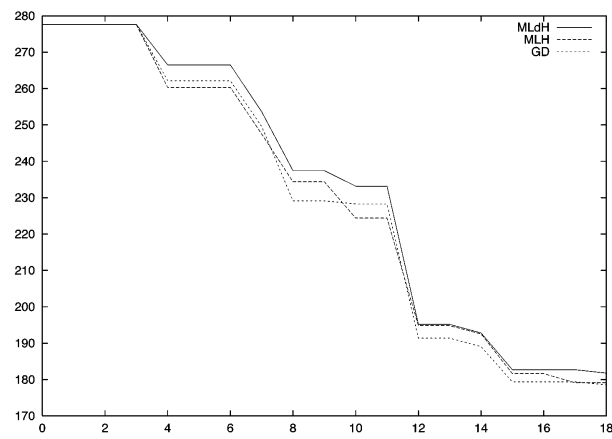

Fig. 2. Evolution of the SSD criterion during first 18 iterations when registering the Lena image, artificially deformed with $2 \times 4 \times 4$ cubic B-spline coefficients and a maximum displacement of about 30 pixels, without multiresolution. The optimizers used were: Marquardt-Levenberg with full Hessian (MLH), Marquardt-Levenberg with only the diagonal of the Hessian taken into account (MLdH), and gradient descent (GD). The deformation was recovered in all cases with an accuracy between 0.1 and 0.01 pixels (see also Section VII).

TABLE I

Relative Times to Evaluate the CRITERION $E$, ITs GRAdiEnt $\nabla E$, AND HESSIAN $\nabla^{2} E$, FOR A VOLUME OF $64 \times 64 \times 17$ VOXXLS APPROXIMATED BY Cubic Splines, as a Function of the SPLINE DEgREe $n_{m}$ USED to Model THE DEFormation AND the Size of the PaRAmeter Grid $n_{c}$. (THE ABsolute Time to Evaluate $E$ Was ABout $1 \mathrm{~s}$ )

\begin{tabular}{c||r|r|r}
\hline$n_{m}$ & 2 & 3 & 3 \\
\hline$n_{c}$ & $3 \times 6 \times 6 \times 6$ & $3 \times 4 \times 4 \times 4$ & $3 \times 6 \times 6 \times 6$ \\
\hline$E$ & 1.0 & 1.4 & 1.4 \\
\hline$E, \nabla E$ & 2.3 & 2.9 & 2.9 \\
\hline$E, \nabla E, \nabla^{2} E$ & 10.9 & 48.7 & 48.8 \\
\hline
\end{tabular}

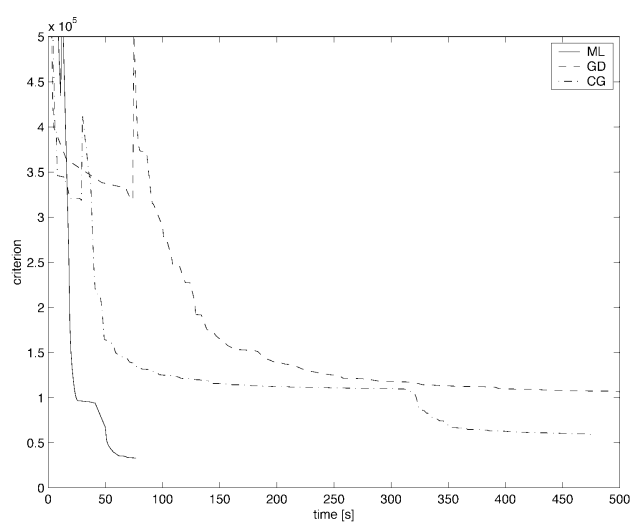

Fig. 3. Comparison of gradient descent (GD), conjugated gradient (CG), and Marquardt-Levenberg (ML) optimization algorithm performances when registering SPECT images with control grid of $6 \times 6 \times 6$ knots. The graphs give the value of the finest-level SSD criterion of all successful (i.e., criteriondecreasing) iterations as a function of the execution time. The abrupt changes are caused by transitions between resolution levels.

Under different constraints, when a small number of parameters is sought, the criterion is smooth, and high precision is needed, the ML algorithm performs the best. This is because its higher cost per iteration is compensated for by a smaller number of iterations due to the quadratic convergence. An example of such a situation is shown in Fig. 3. (See also [57].) Among Marquardt-Levenberg $(\mathrm{ML})$ algorithms, we found the performance to be superior when using the full Hessian. 


\section{B. Multiresolution}

As in [22], we use the multiresolution approach for both the image and deformation models. We start with the coarsest resolution versions of both, and alternatively refine the image and the deformation model every time convergence is reached, until the finest level. The coarse versions of images are generated using a reduction operator (see Section VI-C). Conversely, coarse level solutions are extrapolated to finer levels using an expansion operator (cubic spline interpolation).

\section{SEMI-Automatic Registration}

We realize that although the multiresolution approach leads to a very robust registration algorithm, there are cases when it is mislead by an apparent similarity of features which do not correspond physically. Therefore, we developed an extension of the algorithm which can use expert hints. The hints come in the form of a set of landmarks and are used to gear the algorithm toward the correct solution.

The idea of a hybrid registration algorithm combining both local features (points or lines) with global ones (intensities) has appeared for example in [58], [59 ${ }^{6}$ and others. However, as both [58], [59] use essentially local, nonparametric deformation models, the landmark constraints need to be first interpolated everywhere to serve as an a priori deformation field. This is in contrast with our method which only imposes the landmark information at landmark points where it is really known. Another difference is that thanks to our parametric deformation model the additional overhead is negligible.

The landmark information is incorporated in the automatic process using the concept of virtual springs, tying each pair of corresponding points together. We augment the data part of the criterion $E$ with a term $E_{s}$, corresponding to the potential energy of the springs, and minimize the sum of the two: $E_{c}=E+E_{s}$. The spring term is

$$
E_{s}=\sum_{i=1}^{S} \alpha_{i}\left\|\mathbf{g}\left(\mathbf{x}_{i}\right)-\mathbf{z}_{i}\right\|^{2}
$$

where $S$ is the number of springs, $\alpha_{i}$ are weighting factors corresponding to their stiffnesses, and $\mathbf{x}_{i}$, resp. $\mathbf{z}_{i}$, are the landmark positions in the reference, resp. test images. The spring factors $\alpha_{i}$ control the influence of the particular landmark pairs. We propose to start with all $\alpha_{i}=1.0$ and adjust them experimentally to get the most satisfactory results. We should aim for a compromise between $\alpha_{i}$ too small that does not succeed in making the algorithm to converge to the right solution, and $\alpha_{i}$ too high that forces the solution to a landmark position that is perhaps not sufficiently precise.

As an example, we tried to register an MRI slice from an atlas $^{7}$ with a sample MRI test image. ${ }^{8}$ The atlas is a labeled and annotated collection of images. To identify the same structures in the test image, we register it with the unlabeled version of the atlas. Once the geometric correspondence is established, the

${ }^{6}$ We thank the reviewers for bringing this to our attention.

${ }^{7}$ Courtesy of Harvard Medical School, http://www.med.harvard.edu/ AANLIB/home.html.

${ }^{8}$ We use a proton density MR image from the Visible Human project http:// www.meddean.luc.edu/lumen/meded/grossanatomy/cross_section/index.html. structures and their labels from the atlas can be projected onto the test image. Prior to registration, the histogram of the test image was matched to that of the reference. The unsupervised registration correctly registers some of the structures but misses others; in particular the skull boundary (see Fig. 4). We then identified several landmarks in both images (Fig. 5). Using this minute hint, the semi-automatic algorithm could recover a plausible deformation, even though the landmark information alone (using e.g., thin-plate splines) would not have been enough [38]. We gave the weight 1.0 to all landmarks except the landmark at the bottom left part of the skull which had a weight of 0.2. This made the final positions of the landmarks coincide with the target ones to within about 2 pixel for the least weighted landmark and about 1 pixel for all the others.

Adding the spring term privileges likely solutions based on our a priori knowledge and makes the problem better-posed. The points need not to be image-dependent landmarks. For example anchoring the four corners of the image prevents the solution from degenerating. In this way, the springs play in part the role of a regularization factor.

The landmarks are added when the automatic algorithm cannot solve the problem by itself and an input from a human expert is needed. For this reason, we decided to accept the landmark data as trustworthy and definitive. This is unlike in [58], [59], where the landmarks come from an automatic process, such as iterative closest-point algorithm (ICRP), and therefore cannot be regarded as definitive. However, it is possible to give a certain feedback to the expert, for example the value of the criterion in landmark neighborhoods. This could be also used to reject misplaced landmarks.

\section{IMPLEMENTATION ISSUES}

The purpose of this section is to describe some specific aspects of our implementation. These are mostly independent of the main philosophy of the algorithm but can have a major impact on its performance.

\section{A. Explicit Derivatives}

For the optimization algorithm, we need to calculate the partial derivatives of $E$, as they form the gradient vector $\nabla_{\mathbf{c}} E\left(\mathbf{c}^{(i)}\right)$ and the Hessian matrix $\nabla_{\mathbf{c}}^{2} E\left(\mathbf{c}^{(i)}\right)$. Starting from equation (1), we obtain the first partial derivatives

$$
\frac{\partial E}{\partial c_{\mathbf{j}, m}}=\left.\frac{1}{\|I\|} \sum_{\mathbf{i} \in I_{b}} \frac{\partial e_{\mathbf{i}}}{\partial f_{w}(\mathbf{i})} \frac{\partial f_{t}^{c}(\mathbf{x})}{\partial x_{m}}\right|_{\mathbf{x}=\mathbf{g}(\mathbf{i})} \frac{\partial g_{m}(\mathbf{i})}{\partial c_{\mathbf{j}, m}}
$$

as well as the second partial derivatives

$$
\begin{aligned}
\frac{\partial^{2} E}{\partial c_{\mathbf{j}, m} \partial c_{\mathbf{k}, n}}=\frac{1}{\|I\|} & \sum_{\mathbf{i} \in I_{b}}\left(\frac{\partial^{2} e_{\mathbf{i}}}{\partial f_{w}(\mathbf{i})^{2}} \frac{\partial f_{t}^{c}}{\partial x_{m}} \frac{\partial f_{t}^{c}}{\partial x_{n}}\right. \\
& \left.+\frac{\partial e_{\mathbf{i}}}{\partial f_{w}(\mathbf{i})} \frac{\partial^{2} f_{t}^{c}}{\partial x_{m} \partial x_{n}}\right) \frac{\partial g_{m}}{\partial c_{\mathbf{j}, m}} \frac{\partial g_{n}}{\partial c_{\mathbf{k}, n}} .
\end{aligned}
$$

From (1) defining the SSD criterion, we get $\left(\partial e_{\mathbf{i}}\right) /\left(\partial f_{w}(\mathbf{i})\right)=$ $2\left(f_{w}(\mathbf{i})-f_{r}(\mathbf{i})\right)$ and $\left(\partial^{2} e_{\mathbf{i}}\right) /\left(\partial f_{w}(\mathbf{i})^{2}\right)=2$. The derivative of the deformation function (4) is simply $\left(\partial g_{m}\right) /\left(\partial c_{\mathbf{j}, m}\right)=$ $\beta_{n_{m}}(\mathbf{x} / \mathbf{h}-\mathbf{j})$. The deformation model is linear and all its second derivatives are therefore zero; that is the reason for the 


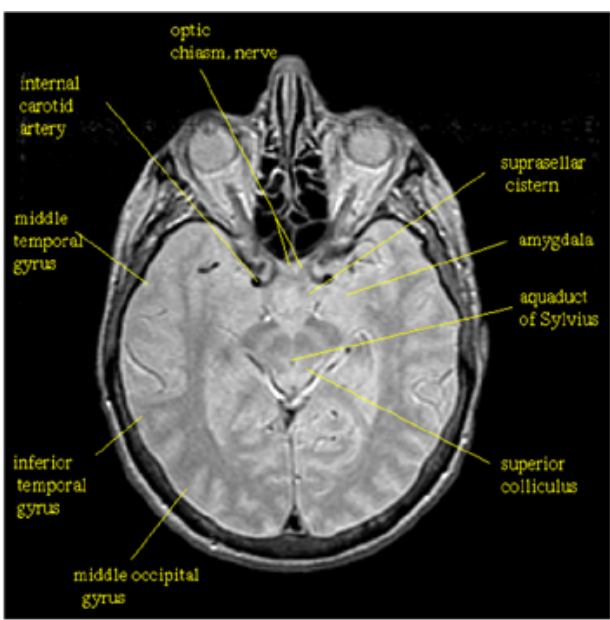

(a)

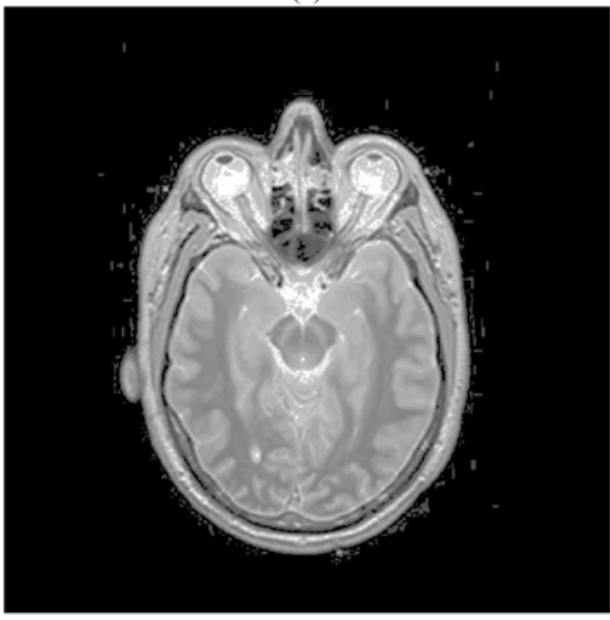

(c)

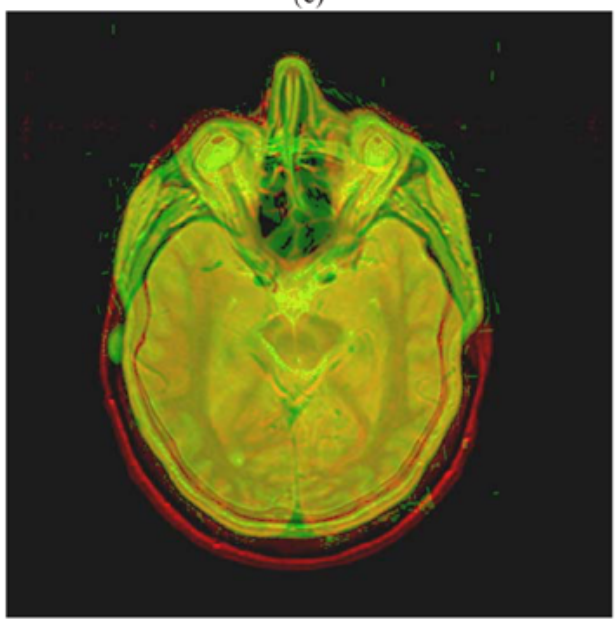

(e)

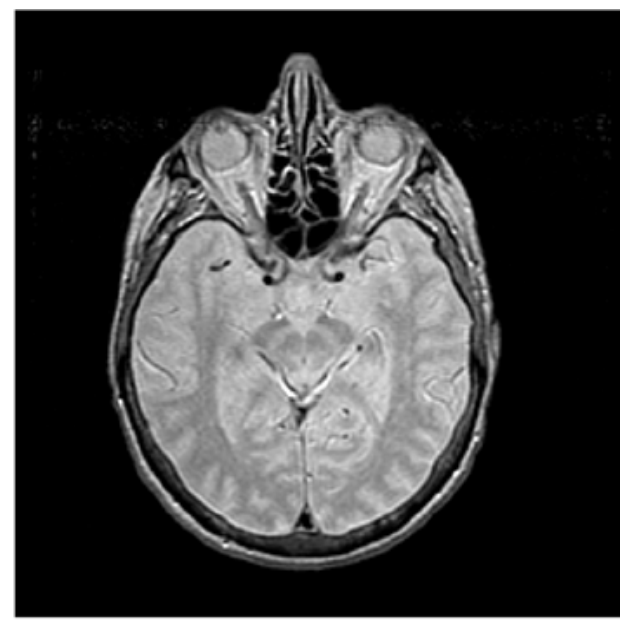

(b)

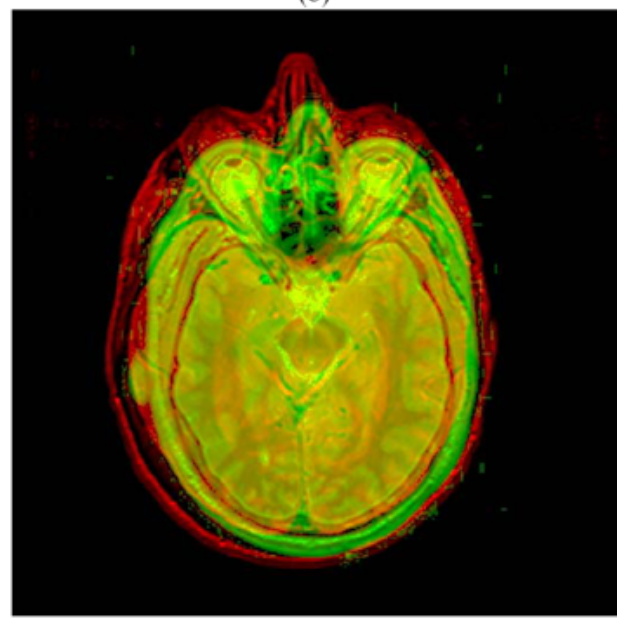

(d)

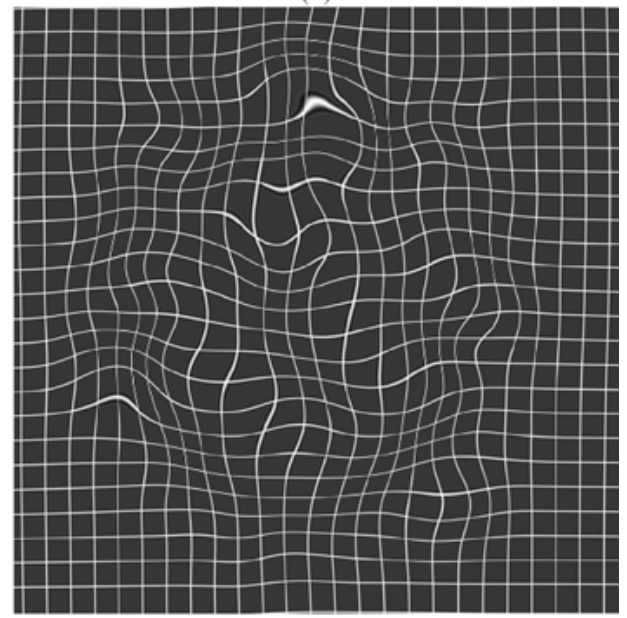

(f)

Fig. 4. Reference MRI proton density brain slice from the atlas (a) with and (b) without labels. The sample test slice of a corresponding region (c). The superposition (in red and green) of the two images (d) before and (e) after the registration. The deformation field (f). Cubic splines were used with knot spacing of $h=32$. The image size was $512 \times 512$ pixels. The difference between images is only partially corrected by the unsupervised registration. Misalignment of several structures is clearly visible.

simplicity of (7). The partial derivatives of $f_{t}^{c}$ in (6) and (7) can be calculated from (2) as a tensor product

$$
\frac{\partial f_{t}^{c}}{\partial x_{m}}(\mathbf{x})=\sum_{\mathbf{k} \in I} b_{\mathbf{k}} \beta_{n}^{\prime}\left(x_{m}-k_{m}\right) \prod_{\substack{l=1 \\ l \neq m}}^{N} \beta_{n}\left(x_{l}-k_{l}\right) .
$$

Second-order partial derivatives of $f_{t}^{c}$ are obtained in a similar fashion.

The Marquardt-Levenberg approximation of the Hessian assumes that the term $\left(\partial e_{\mathbf{i}}\right) /\left(\partial f_{w}(\mathbf{i})\right)$ is negligibly small or that it sums to zero on average, which justifies omitting this term from (7); see [43]. Another simplification is to consider only 




(a)

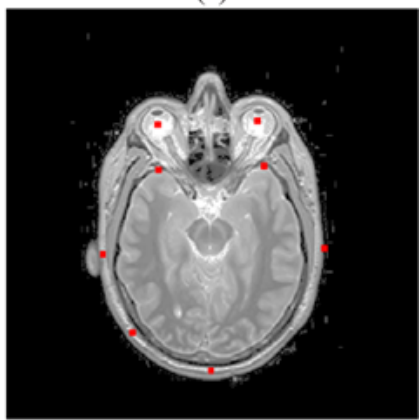

(b)

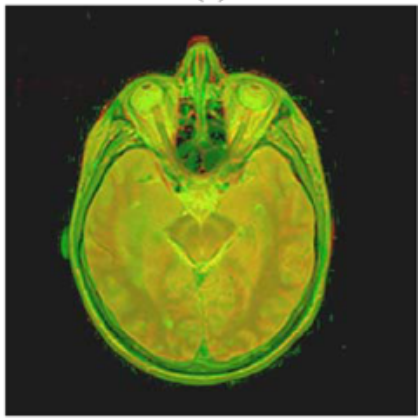

(c)

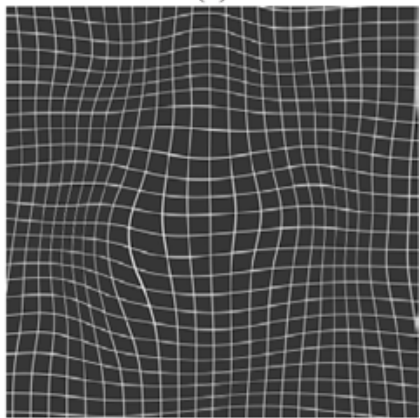

(d)

Fig. 5. Reference (a) and test (b) images with superimposed landmarks (in red). The superimposed images after registration using the semi-automatic algorithm (c) and the deformation field found (d). Corresponding anatomical structures are well identified; the alignment is clearly superior to that in Fig. 4.

diagonal terms $\partial^{2} E / \partial c_{\mathbf{j}, m}^{2}$. Obviously, this diagonal Hessian approximation only makes sense if the basis functions $\varphi_{\mathbf{j}}$ do not overlap too much. This is another argument for the B-spline model.

\section{B. Gradient Calculation as a Convolution}

Similarly to the case of evaluating the deformation $\mathrm{g}$, the use of an integer step size $\mathbf{h}$ leads to computational savings here too.
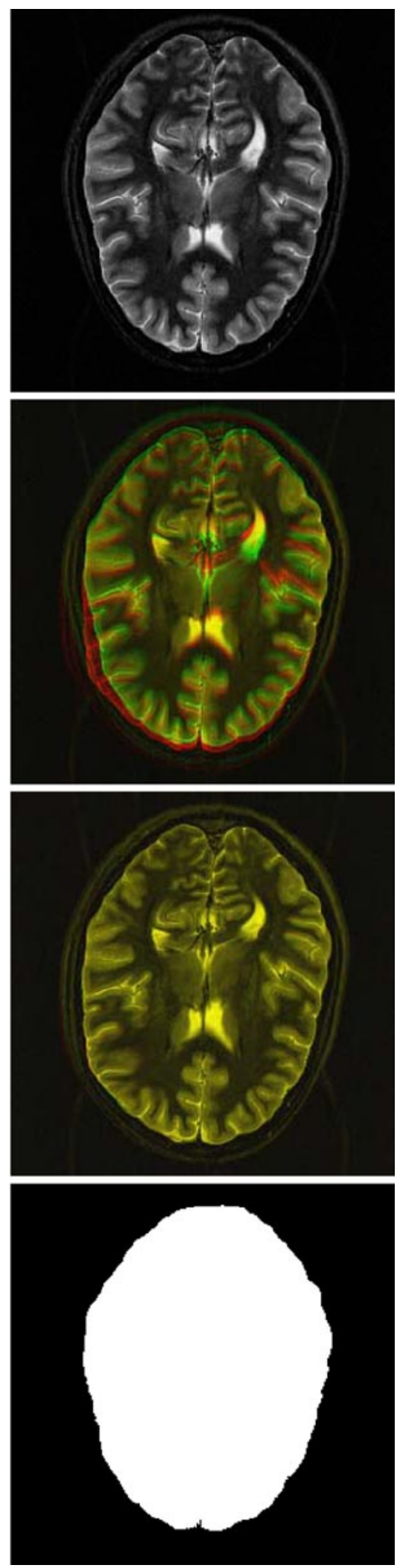

Fig. 6. From top to bottom: The original slice of anatomical MRI brain image, original superimposed over the true deformation, the recovered deformation versus the true deformation, and the mask used to calculate the warping index (bottom left).

The expanded expression for $(\partial E) /\left(\partial c_{\mathbf{j}}, m\right)$ can be transformed into a discrete separable convolution $\left\{(\partial E) /\left(\partial c_{\mathbf{j}}, m\right)\right\}_{\mathbf{j}}=$ 

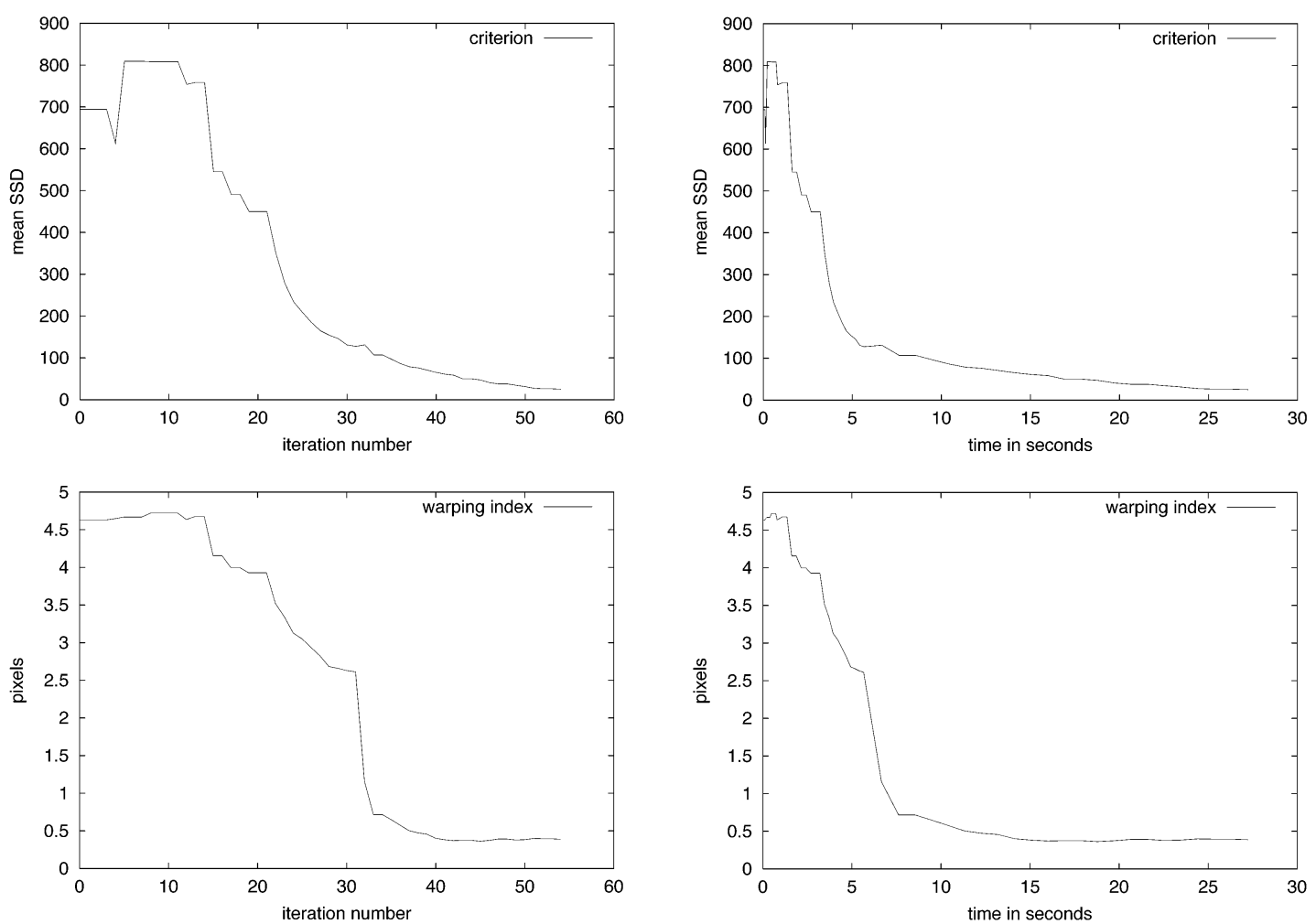

Fig. 7. Evolution of the optimization process. The left column displays the evolution with respect to the number of iterations, while the right column represents the same quantity respect to time. The first row shows the SSD criterion $E$, the second row the warping index $\varpi$. The step changes correspond to the changes in the model and image resolutions. We observe good correlation between all four graphs.

$\sum_{\mathbf{i}} w(\mathbf{i}) b(\mathbf{j} \cdot \mathbf{h}-\mathbf{i})=(w * b)_{\downarrow \mathbf{h}}$, where we have substituted $w$ for the first two factors in $(6), b(\mathbf{q})=\beta_{n_{m}}(-\mathbf{q} / \mathbf{h})$, and $\downarrow \mathbf{h}$ indicates downsampling as defined by the formula, with elementwise multiplication $\mathbf{j} \cdot \mathbf{h}$. The convolution kernel $b$ is separable and the convolution can be calculated as a sequence of $N$ unidimensional convolutions $\left(\left(w * b_{1}\right)_{\downarrow h_{1}} * \cdots b_{n}\right)_{\downarrow h_{N}}$. Because of the downsampling, calculating one output value at step $k$ consists of a scalar product with a filter $b_{k}$ of length $\left(n_{m}+1\right) h_{k}$ and shifting this filter by $h_{k}$.

\section{Multiresolution Spline Representation}

To deploy the multiresolution strategy (see Section IV-B), we need to specify expansion and reduction operators. We will use the same approach for both the deformation model and the image model. The expansion can be performed exactly; we choose to do optimal reduction in the $L_{2}$ sense [60]. Both expansions and reductions can be performed efficiently using FIR and recursive IIR filters. To cope with the finite extent of our signals, we put extra B-splines outside the interval of interest. This allows for complete control of the signal within the interval of interest; see [38] for details.

\section{Fast Spline Calculations}

It is essential to take full advantage of the properties of splines. First, specialized routines are used to calculate the values of a B-spline of a specific order using a minimum number of operations. Second, as we are using tensor products of B-splines as our basis functions, many operations can be

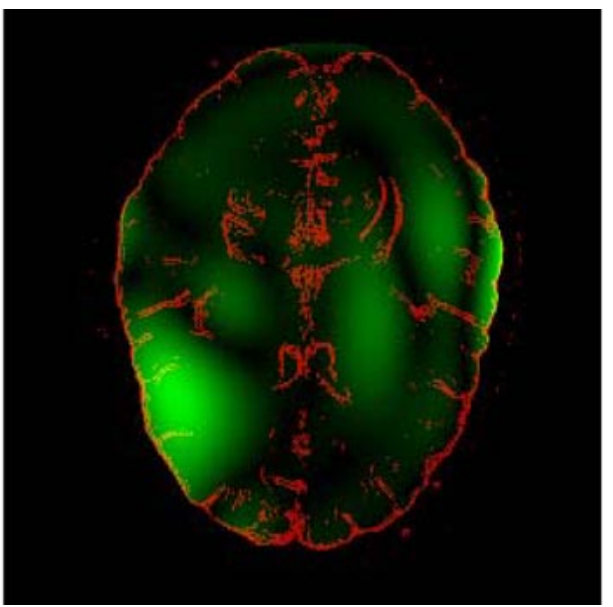

Fig. 8. Geometrical error after registration (green) with superposed contours of the original MRI image (red). The maximum (green) intensity corresponds to an error of 1.5 pixels.

performed in a separable fashion, reducing the complexity of operations from $O\left(k^{N}\right)$, where $N$ is the number of dimensions and $k$ the size of the data, to $O(k N)$. This is the case for the prefiltering step required to find the B-spline coefficients, and also for the interpolation of values of a function given by its B-spline coefficients. Third, the compact support of B-splines simplifies many of the infinite sums in the expressions given earlier, reducing them to sums over just a small number of elements. 

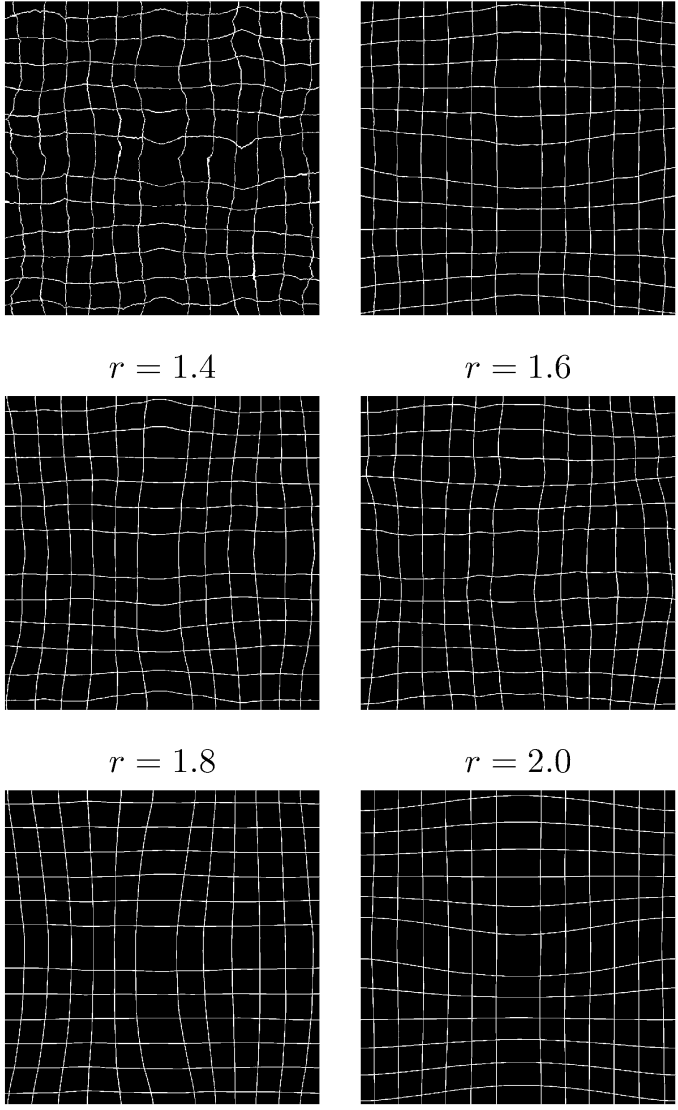

$r=2.2$

$r=2.4$

Fig. 9. Examples of randomly generated fractal-like deformations for various Sobolev exponents $r$. Observe how the deformation gets smoother with increasing $r$.

\section{E. Stopping Criterion}

To get a fast optimization algorithm, particular attention has to be paid to the stopping criterion. This holds for both GD and ML algorithms. Classically, the relative and absolute improvement of the criterion value is compared with a fixed threshold [43]. For our class of problems, we found it to be advantageous to base the stopping criterion on the changes $\Delta \mathbf{c}$ of parameter values. We stop when the step size falls below an a priori given threshold $\varepsilon$. The size of a step that fails gives an indication of the accuracy of the result and is therefore easy to set. Typically, we would use the threshold of $\varepsilon=10^{-1} \sim 10^{-3}$ pixels for the finest level an slightly more for coarser levels, as there is usually not enough details and coherence between levels.

\section{F. Masking}

A substantial gain in speed comes from considering only important pixels when calculating the data criterion (1) and its derivatives. It is possible to determine an a priori mask of significant pixels, for example 10 50\% of the total number of pixels, and to consider only those pixels in subsequent calculations. The contributions of individual pixels to the change of the criterion is directly proportional to the amplitude of the directional derivatives at the respective points; see (6). Therefore, a reasonable strategy is to construct the mask by thresholding the gradient of the image at each multiresolution level.

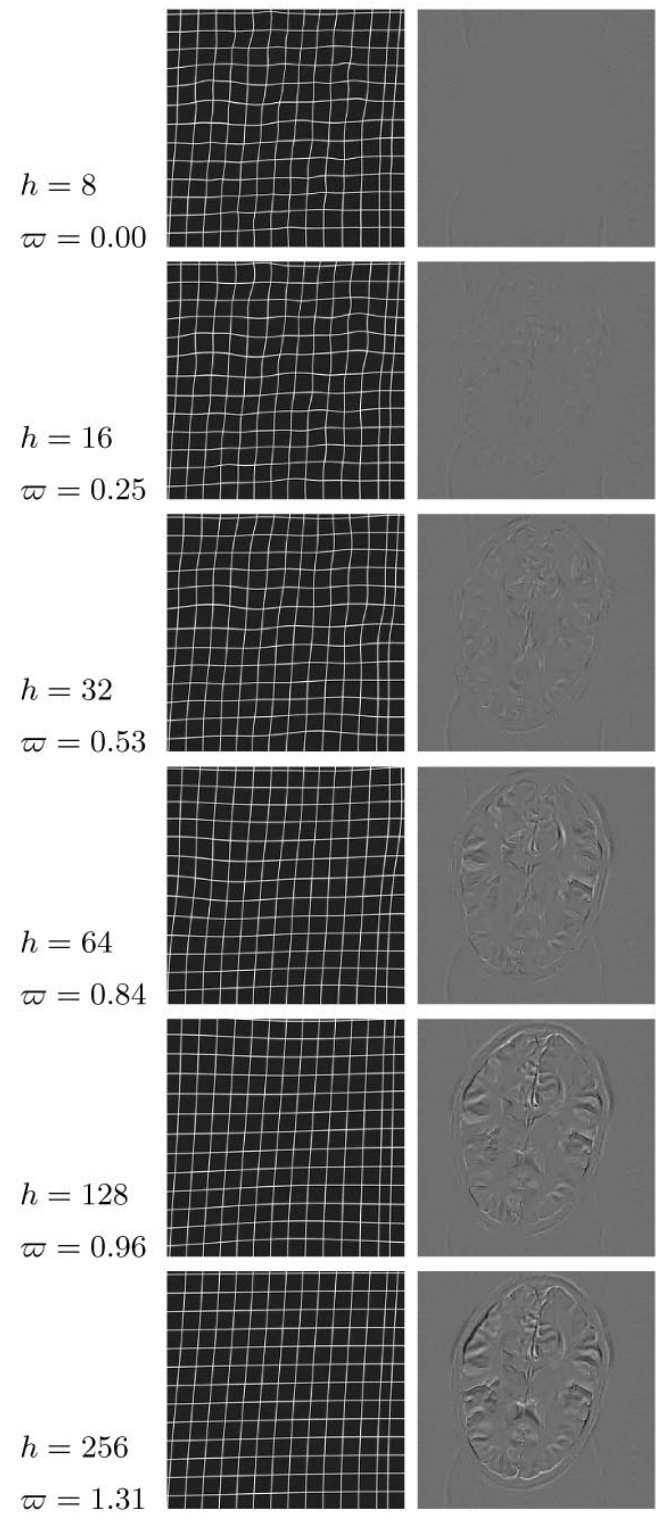

Fig. 10. Deformation recovered using progressively smaller (coarser) deformation spaces (left column), and the corresponding residual error (right column). The knot spacing $h$ and warping index $\varpi$ are shown. Cubic splines were used.

\section{EXPERIMENTS}

This section presents a series of experiments in a controlled environment to assess the accuracy, speed, and robustness of our algorithm. We show the SSD criterion (1) we minimize, and also a warping index $\varpi=\sqrt{1 /(\|R\|) \sum_{\mathbf{i} \in R}\left\|\mathbf{g}(\mathbf{i})-\mathbf{g}^{*}(\mathbf{i})\right\|^{2}}$; that is, the mean geometric error between the true and the recovered deformation. The mean is only calculated over a region $R$, the part of the image containing useful data (object); an example of a region can be seen in Fig. 6, bottom left.

\section{A. Registration of MRI Brain Slices}

To illustrate the behavior of the algorithm, we show its performance when recovering a known deformation of a two-dimensional (2-D) slice of an anatomical spin-echo MRI volume 


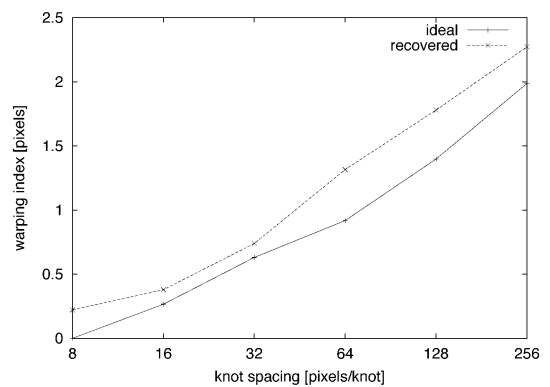

Fig. 11. Ideal (best achievable) versus attained warping index when recovering the randomly generated hierarchical deformation $(r=2)$ applied on a MRI image (see Fig. 10) as a function of the search space.

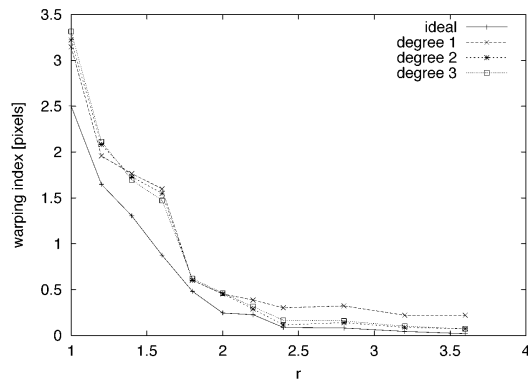

Fig. 12. Final geometric error as a function of the smoothness (regularity) of the deformation (high $r$ corresponds to smoother functions) and the spline degree of the deformation model. The "ideal" warping index corresponds to the projection into the cubic spline space. The values shown are arithmetic means of 60 experiments. The standard deviation of the warping index $\varpi$ is about 0.25 pixels.

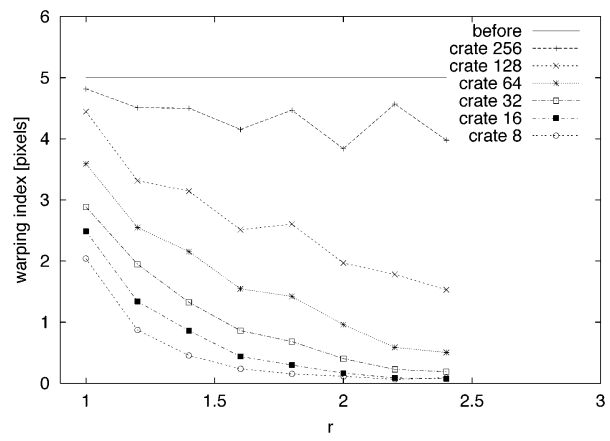

Fig. 13. Final geometric error as a function of the grid spacing $h$ (crate) in pixels and the smoothness of the deformation (high $r$ corresponds to smoother functions). The values shown are arithmetic means of 60 experiments.

of the brain. ${ }^{9}$ We use here artificially deformed images because the knowledge of the ground truth permits us to better judge the performance of the algorithm.

The original image of size $256 \times 256$ pixels is shown in Fig. 6 , top left. We use a cubic spline control grid with one knot for every 32 pixels. We warp the image with a deformation belonging to the warp space and consisting of displacements up to 15 pixels ( 1 pixel corresponds to approximately $0.9 \mathrm{~mm}$ ). The warped image is superimposed on the original in Fig. 6, top right. Then the automatic registration algorithm is run. The stopping threshold is set to 0.5 pixels for all levels except the last, where we set it to 0.1 pixels. The recovered deformation was used to warp again the original image. Its warped version

${ }^{9}$ First author's brain. Images courtesy of Arto Nirkko from Inselspital Hospital, Bern, Switzerland.

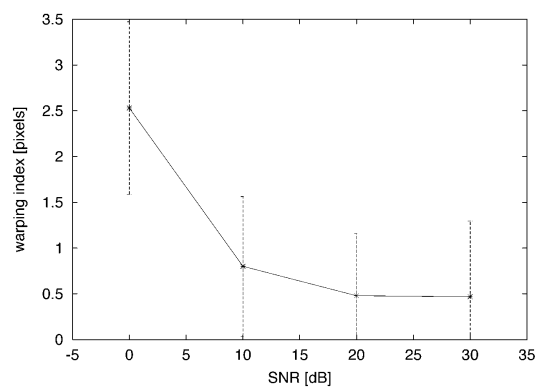

Fig. 14. Final geometric error as a function of the SNR. The values shown are arithmetic means of 60 experiments. Error bars indicate one standard deviation.
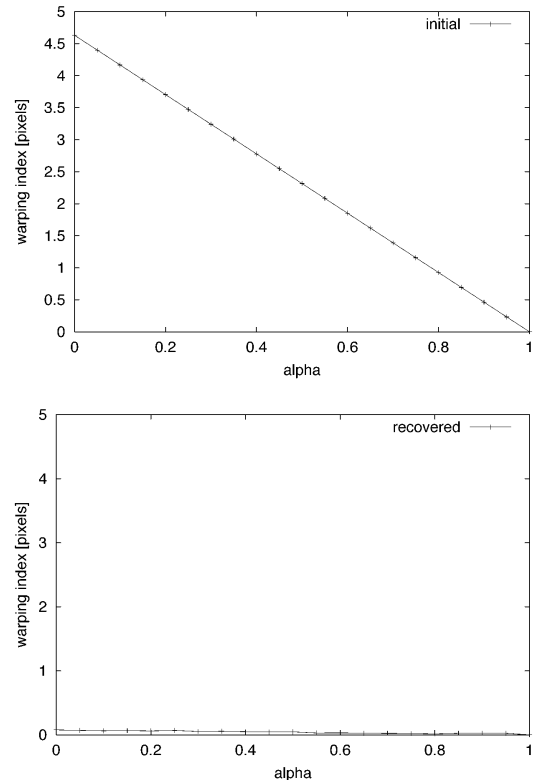

Fig. 15. Warping index of the deformation used as a starting point (top) and of the recovered deformation (bottom). The initial guess (starting point) varies from identity $(\alpha=0)$ to the true deformation $(\alpha=1.0)$.



Fig. 16. Scatter plot of the dependence between the original and final warping indexes. The data is based on 142 experiments.

is shown superimposed on the image warped with the true deformation in Fig. 6, bottom right. We note that the deformation was well recovered with no perceptible difference.

The spatial distribution of the resulting geometrical error is shown in Fig. 8. The maximum error is about 1.5 pixels, while the mean geometric error (warping index $\varpi$ ) over the total of the brain is about 0.4 pixels. We generally observe that the error is concentrated in areas with little detail in the image. Other, high-contrast regions such us edges are resolved much more 


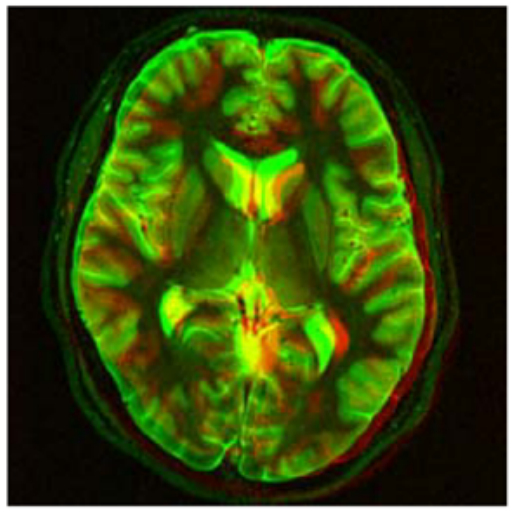

(a)



(b)



(c)

Fig. 17. The superposition of the slices of anatomical MRI images before the registration (a), after the registration (b), and the resulting deformation field (c). Quadratic splines were used with knot spacing of $h=64$.

precisely than indicated by the value of $\varpi$, often with subpixel accuracy. On the other hand the agreement in the zones with low-contrast will be worse and often only coincidental, since there is little or no information to guide the algorithm.

The evolution of the optimization can be studied from the graphs in Fig. 7. We observe the steady and correlated descent of the observable criterion being optimized $(E)$ and of the warping index $(\varpi)$, the quantity measuring the quality of the registration. The abrupt changes in the curves are caused by the transitions between levels of the multiresolution progression; they are small thanks to the accuracy of the spline model.

Note that the final values of both $E$ and $\varpi$ depend strongly on the preset stopping threshold, which in turn influences the optimization time. The threshold value is a subjective compromise between the accuracy and computation time. It is perfectly possible to stop optimizing only after $7 \mathrm{~s}$ and skip the finest resolution level altogether, if the precision of $\varpi=0.7$ pixels is acceptable. On the other hand, after about 4 more min of iteration, the error $\varpi$ descends to less than $10^{-4}$ pixels. However, in the authors' opinion, such super subpixel accuracy is almost never achievable on real images, because of the noise and the unknown characteristics of the acquisition process.

\section{B. Deformation Generator}

We have implemented a fractional wavelet based random deformation generator. It yields deformations with a prescribed smoothness (regularity), characterized by a Sobolev exponent $r$-the maximum number of (fractional) derivatives in the $L_{2}$ sense. This is guaranteed if the Fourier transform decreases asymptotically at least as $1 / \omega^{r+0.5}$. We express the random displacement $\mathbf{g}(\mathbf{x})-\mathbf{x}$ in an orthogonal wavelet basis. We use orthonormal symmetric fractional B-spline wavelets [61], [62] of degree $\alpha=r-0.5$, which have precisely the desired regularity and Fourier decay at infinity. We let the wavelet coefficients $\vartheta_{j, k}$ be random (zero mean, independent, and normally distributed) with standard deviation decreasing as $2^{-r j}$, where $j$ denotes the scale. This makes the Fourier spectrum of the displacement decrease as required over the whole frequency range and ensures that the (mean) displacement belong to the Sobolev space $W_{2}^{r}$ [63].
To obtain corresponding 2-D deformation fields, we use separable 2-D wavelet transforms with the same basis functions and the same decrease of amplitude of the coefficients in each component as in the 1-D case. We can observe in Fig. 9 how the deformation gets progressively more smooth and regular with increasing $r$.

\section{Out-of-Space Deformation}

The true deformation is not guaranteed to lie in the space where we are looking for it and can therefore never be recovered exactly. The associated error is called an approximation error. We performed various experiments to compare the approximation error with the overall registration error. We generated a random hierarchical deformation using the wavelet methodology from the previous section (with $r=2$ ) and projected it into the space with knot spacing $h=8$. We deformed the MRI image (Fig. 6) with this deformation and tried to recover it in spaces with knot spacings $h=8 \sim 256$. Fig. 10 shows the recovered deformations and the residual differences between the reference image and the warped test images for different values of the knot spacing $h$. We observe that the deformation can be recovered almost completely when we search in the correct space $(h=8)$; important errors arise when we search in different, coarser spaces. Ultimately, for $h=256$, we can express only deformations close to affine, which is obviously not enough to capture all the details of the true deformation.

We now compare the error that our algorithm yields with the smallest error it could possibly achieve, given the search space. To find the best achievable approximation of some deformation, given the knot spacing and spline degree, we will use the fact that the warping index is in fact the $\ell_{2}$ (Euclidean) distance. Therefore, the best approximation is an orthogonal projection of the deformation onto the search space which can be calculated easily.

The warping index resulting from the registration process is compared with the best achievable one in a given space in Fig. 11. We see that although the ideal values are not attained, the difference is within the range of half a pixel. In real situations, the true deformation space is not known. However, thanks to the good approximation properties of splines, we can reasonably expect that by using a sufficiently small value of $h$, we can reduce the approximation error to an acceptable value. 


\section{Choosing the Spline Degree}

The choice of the spline degree for the image and deformation models is a trade-off between the accuracy and speed. Here too we generated hierarchical random deformations (see Section VII-C) with varying smoothness and applied them on the MRI image. We recover the deformations in spline spaces with grid spacing $h=32$ pixels for linear, quadratic, and cubic spline deformation models, with $\varepsilon=0.01$ pixels. We observe (see Fig. 12) that higher order splines perform better, while the difference between linear and quadratic is much more important than between quadratic and cubic splines. The sample registration times were $20.1 \mathrm{~s}, 26.7 \mathrm{~s}$, and $48.9 \mathrm{~s}$, for linear, quadratic, and cubic splines, respectively. This indicates that to use quadratic splines for the deformation model might be a good compromise between approximation properties and speed. Note that the task of recovering fastly changing deformations is doubly difficult, as they cannot be represented well by the deformation model and they do not have a pronounced effect on the image because of its lack of details at small scale in many regions. Note also that as the deformation gets smooth, the geometric error of the recovered deformation gets almost as small as the minimum achievable error.

\section{E. Choosing the Grid Spacing}

Thanks to the properties of our deformation model and the optimization algorithm, the grid spacing and thus the number of parameters influences the execution time only mildly. Therefore, the main criterion for choosing the grid spacing $h$ should be the estimated intrinsic resolution (smoothness) of the deformation to be recovered. A control grid that is too coarse is not able to express the deformation in all details. On the other hand, too fine a control grid is overcompensating for true image differences and noise. The effect of the grid spacing is less pronounced for smoother deformations; see Fig. 13.

\section{F. Noise Dependence}

We added various levels of noise to the test images (i.e., after the warping has been performed) to demonstrate the influence of the SNR (signal to noise ratio) on the registration results. We used 60 random deformation with $r=2.0$, cubic splines with knot spacing $h=32$, and stopping criterion $\varepsilon=0.01$. We observed that for SNR better than $10 \mathrm{~dB}$, the influence of the noise is very small (Fig. 14).

\section{G. Starting Point}

The following experiment evaluates the robustness of the algorithm with respect to the starting point. Here, we tried to recover the deformation from Section VII-A (MRI images) optimizing only at the finest level. We linearly varied the starting point of the optimizer between identity and the true deformation and observed the attained warping index $\varpi$ for a stopping threshold of $\varepsilon=0.01$ pixels. Fig. 15 shows the warping index of the deformation used as a starting point and the warping index of the recovered deformation. We observe that although the final result does depend on the starting point, it is most likely only the influence of the stopping criterion. The algorithm therefore proves to be very robust, even without the help of a multiresolution: it converged in all cases to the desired solution. On the

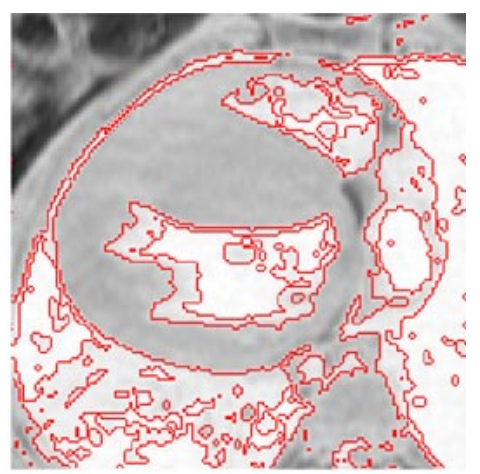

(a)



(b)

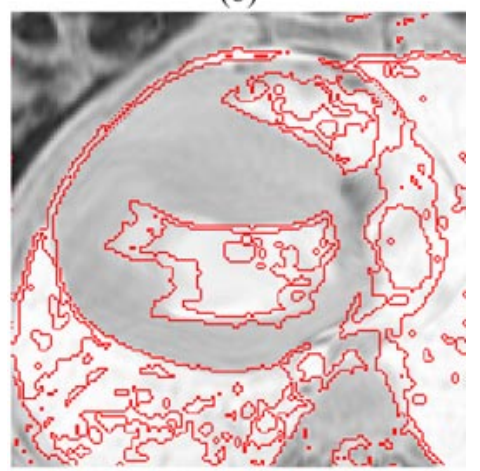

(c)

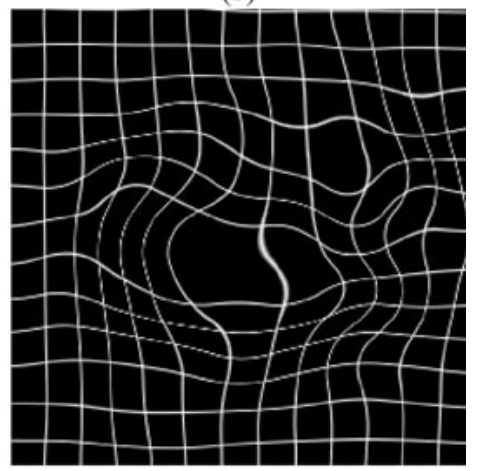

(d)

Fig. 18. Reference MRI image from a heart sequence with superimposed contours (a). The same contours over another image (the test image) from the same sequence before the registration (b) and after (c). The deformation field (d). Quadratic splines were used with knot spacing of $h=64$, image size was $256 \times 256$ pixels.

other hand, the elapsed time and the number of iterations differed significantly, from 2 iterations when starting from the true solution, to several hundreds when starting from identity. 
6
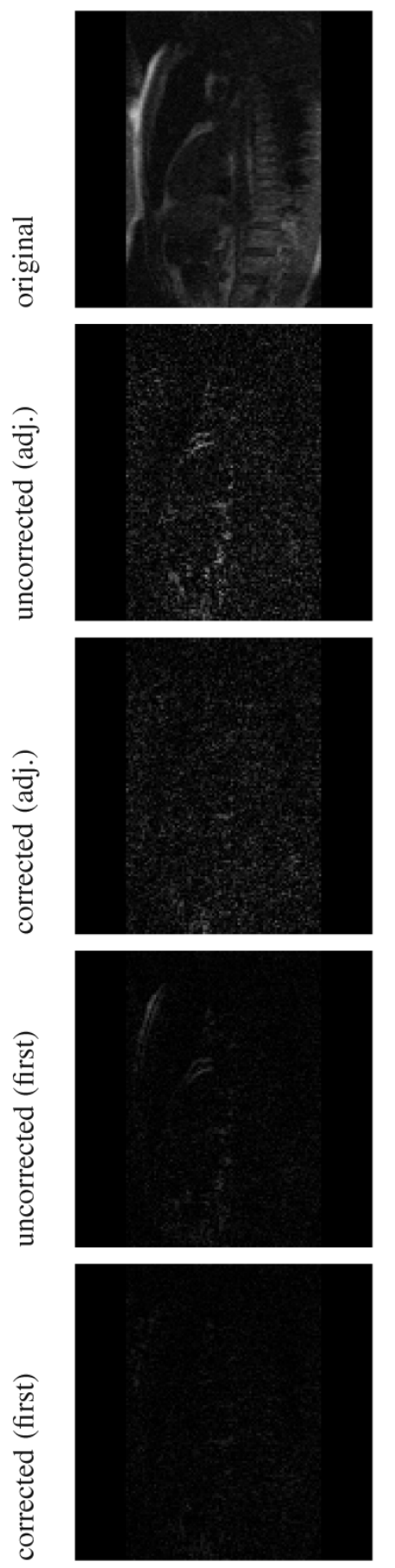

9
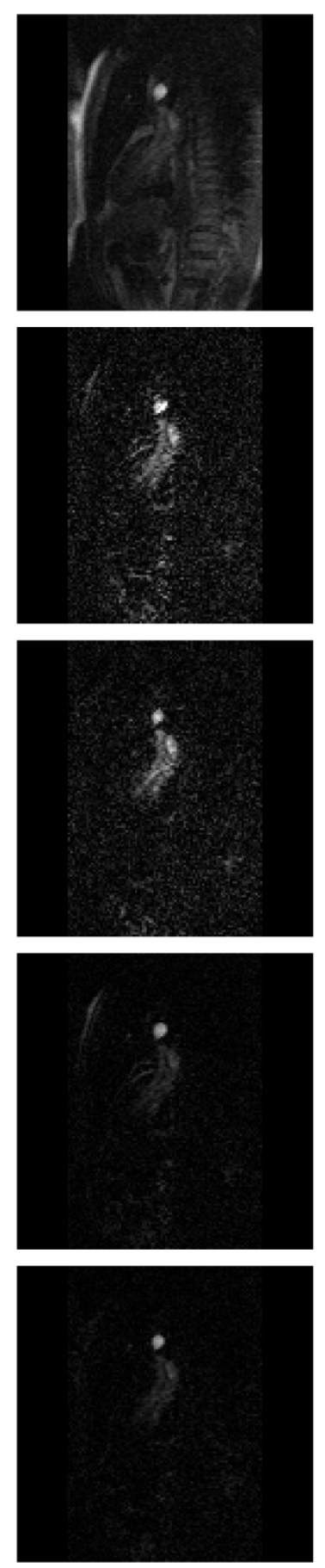

11
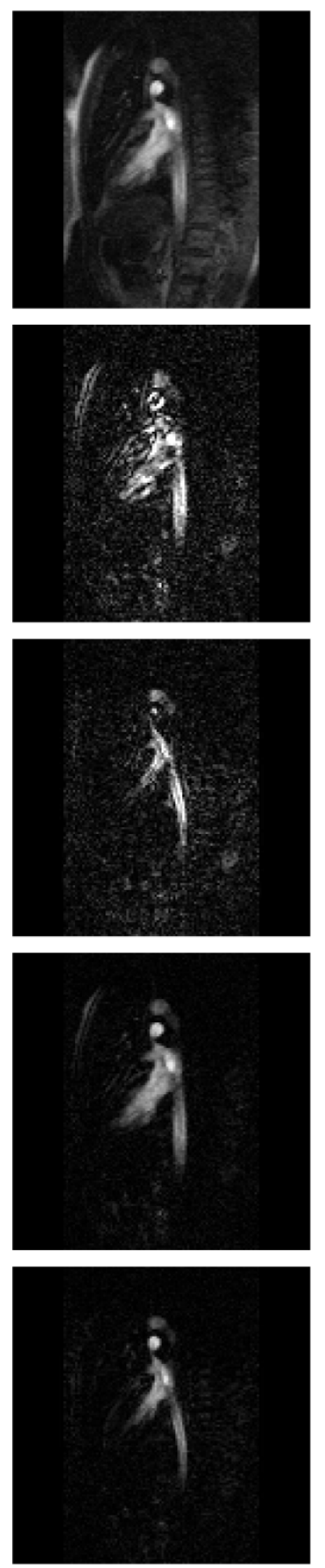

14
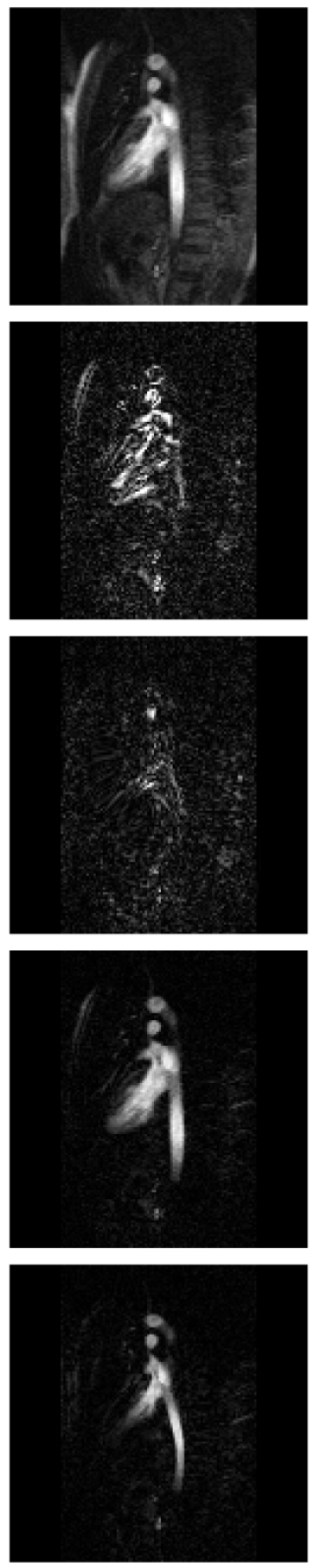

Fig. 19. First line presents original images number $6,9,11$, and 14 from a sequence of originally 60 images of myocardical perfusion MRI. The second line presents the difference images between the original images and their immediate predecessors; movement artifacts can be clearly seen. On the third line you can see the difference images from the motion corrected sequence using our algorithm; the movement artifacts are significantly reduced. The same effect is also visible comparing the differences of the sequence images with the first image of the sequence on the original (fourth line) and corrected (fifth line) sequences.

\section{H. Statistical Distribution of Errors}

To evaluate the behavior of the algorithm on a larger set of test cases, we generated a series of random hierarchical deformations (see Section VII-B), warped the MRI slice with them, and applied our registration algorithm to recover the deformation. We used the stopping threshold $\varepsilon=0.01$ pixels and a warping space which contained the deformation. We then compared the warping index corresponding to the recovered deformation with the initial warping index, that is, the distance between the true deformation and identity. In Fig. 16 we present the scatter plot describing the relation between the initial and final warping indexes. We observe that the algorithm gives results with accuracy consistently better than 0.1 pixels. 


\section{Experiments With Real Data}

We applied our algorithm to various problems involving medical images of several modalities. We developed a registration procedure for ECDf ${ }^{10}$ and Xenon inhalation SPECT images [64] in the view of atlas creation [37]. Fig. 17 shows the resulting alignment obtained after registering two slices of anatomical (spin-echo) MRI images of two different subjects. ${ }^{11}$

To further illustrate the use of our algorithm, we present registered MRI images from a heart beat sequence; ${ }^{12}$ see Fig. 18. The extracted deformation field can be used to extract trajectories of various points in the heart which is important for diagnostic purposes. Analyzing this field also permits the determination of the velocity and derived parameters, such as the accumulated displacement, and strain. We also analyzed standard 2-D ultrasound sequences of the heart [65]. The algorithm proved to be robust to the occasional change of structure (topology) due to the underlying 3-D nature of the true movement. ${ }^{13}$

Another technique for assessing cardiac performance is myocardial perfusion by MRI [66], [67]. A sequence of MRI images $^{14}$ is acquired with at high speed to assess the diffusion of the agent. A role of the registration is to compensate for the (heart) motion to provide the time profiles of the intensities at each tissue point. The profiles are subsequently analyzed to yield the physical (absorption) parameters of the tissue. Fig. 19 shows a few selected images of the sequence. It also shows differences between images; we observe a significant amount of motion artifacts. Most of these artifacts are compensated for in the corrected sequence, where each of the images was registered with (and warped toward) its already corrected predecesor. Ideally, the corrected sequence should appear static, except for the movement of the agent. In this application, a number of virtual springs with carefully chosen weights was used, to make the deformation compensate for the movement of the tissues, but not for the movement of the contrast agent.

Let us end with a 3-D example: the registration of two computer tomography (CT) head volumes. ${ }^{15}$ Due to the large size of the original volumes $(512 \times 512 \times 45$ voxels $)$, it was impractical to perform the registration directly. We chose instead to perform the registration on reduced volumes $(128 \times 128 \times$ 45) which took about $10 \mathrm{~min}$ to complete ${ }^{16}$ with the control knots placed every $8 \times 8 \times 8$ voxels and stopping threshold of $\varepsilon=0.01$ pixels. We then interpolated this deformation to the original volume size. ${ }^{17}$

\footnotetext{
${ }^{10}$ ECD (Technetium Ethylene Cysteine Diethylester) is a radioactively marked intravenously injected agent.

${ }^{11}$ Images courtesy of Arto Nirkko, Inselspital Hospital, Bern, Switzerland.

${ }^{12} \mathrm{LECB}, \mathrm{NIH}, \mathrm{http}: / / \mathrm{www}-$ lecb.ncifcrf.gov/flicker/.

${ }^{13}$ Analyzing directly 3-D ultrasound heart sequences would avoid this problem. However, 3-D heart sequence acquisitions are much more rare in the clinical use.

${ }^{14}$ Courtesy of J.-P. Vallée, Unité d'imagerie numérique, University Hospital, Geneva, Switzerland.

${ }^{15}$ Images courtesy of Philippe Thévenaz, EPFL, Lausanne, Switzerland. The images were acquired using the same machine and the same protocol, but not preregistered.

${ }^{16}$ On a $700 \mathrm{MHz}$ Pentium based computer.

${ }^{17}$ Registering directly the undecimated volumes on the same computer takes about $3 \mathrm{~h}$ with very minor increase in quality as relatively smooth deformations are sought. We are currently working on a optimized reimplementation of the algorithm that should reduce these times considerably.
}


Fig. 20. Axial, sagital, and coronal views of the two CT brain volumes (one in red, second one in green) after the registration.
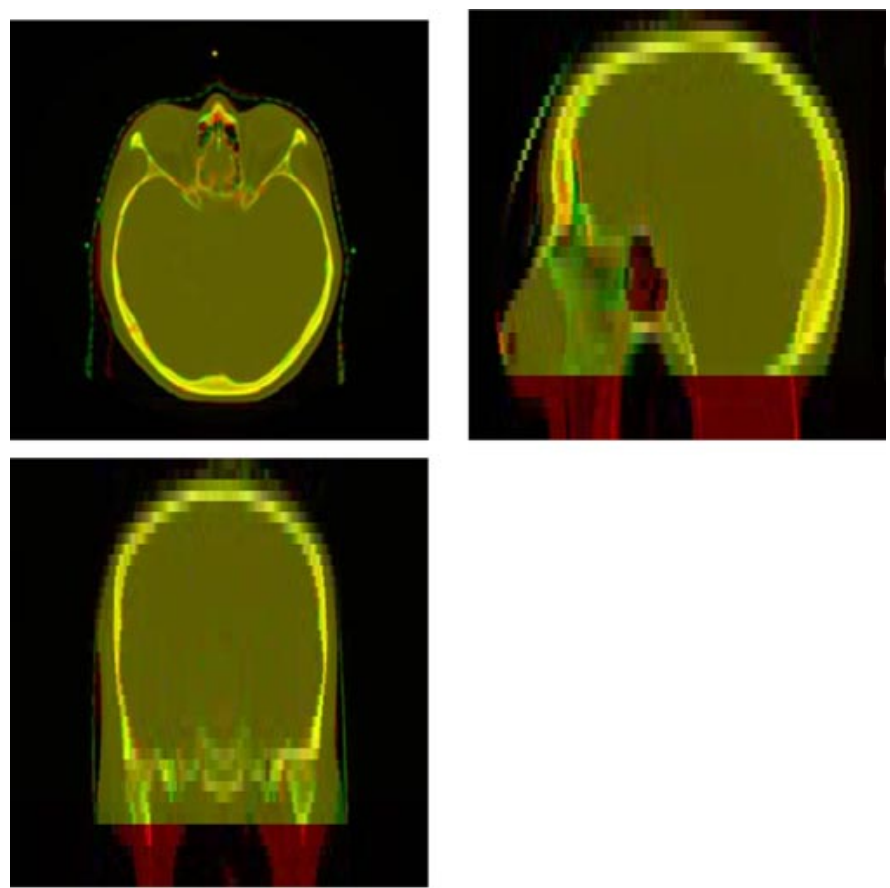

Fig. 21. Axial, sagital, and coronal views of the two CT brain volumes (one in red, second one in green) after the registration. The volumes are aligned, and the large and medium-scale differences were compensated by the registration. This permits to identify more subtle differences.

We observe that it is difficult to do any meaningful comparison of the volumes prior to registration; see Fig. 20. However, once the registration is performed, even small differences are clearly apparent (Fig. 21). Moreover, the deformation field itself can provide valuable quantitative information about the relative sizes and shapes of various parts of the anatomy from the two volumes. Note that the control grid spacing must be adapted to 
the task at hand because it influences the amount of differences compensated for by the registration and warping.

\section{CONCLUSIONS}

We developed a fully automatic elastic registration algorithm. We extended the idea from [22] to multidimensional data, and streamlined the algorithm to accelerate it. We designed a new step-prediction formula for the gradient descent algorithm and showed its efficiency for our application. A double multiresolution strategy brings speed and robustness and additionally eliminates the need for an initial rigid registration as the coarse grid deformation itself plays this role.

We introduced the concept of virtual springs, yielding a semiautomatic registration method, capable of using expert hints in the form of landmarks to solve particularly difficult problems where the fully automatic algorithm may be mislead. This is a powerful combination of the ideas of manual landmark registration and the pixel-based registration using splines.

We applied the algorithm to a wide range of artificially generated problems involving deformations with varying smoothness applied to anatomical MRI images to demonstrate the algorithm's speed, robustness, and accuracy. Furthermore, we presented several medical applications using various image modalities.

We believe that by producing a specialized program taking advantage of a specific configuration, the run time can be decreased by an additional factor of 2 to 10 . This will enable truly interactive operation of automatic and semi-automatic elastic image registration with numerous applications in medicine, biology, and any other field where deformed images need to be compared.

\section{ACKNOWLEDGMENT}

The authors are grateful to Dr. P. Thévenaz for helpful discussions.

\section{REFERENCES}

[1] L. Brown, "A survey of image registration techniques," ACM Comput. Surv., vol. 24, no. 4, pp. 326-376, Dec. 1992.

[2] R. Bajcsy and S. Kovačič, "Multiresolution elastic matching," Comput. Vis., Graph., Image Process., vol. 46, pp. 1-21, 1989.

[3] F. Bookstein, Morphometric Tools for Landmark Data: Geometry and Biology. Cambridge, U.K.: Cambridge Univ. Press, 1997.

[4] A. Mitiche and P. Bouthemy, "Computation and analysis of image motion: A synopsis of current problems and methods," Int. J. Comput. Vis., vol. 19 , no. 1 , pp. 29-55, 1996

[5] R. Szeliski and H.-Y. Shum, "Motion estimation with quadtree splines," IEEE Trans. Pattern Anal. Machine Intell., vol. 18, pp. 1199-1207, Dec. 1996.

[6] A. Spinei, D. Pellerin, and J. Hérault, "Spatiotemporal energy-based method for velocity estimation," Signal Process., vol. 65, pp. 347-362, 1998.

[7] P. Moulin, R. Krishnamurthy, and J. Woods, "Multiscale modeling and estimation of motion fields for video coding," IEEE Trans. Image Processing, vol. 6, pp. 1606-1620, Dec. 1997.

[8] J. Weese, G. P. Penney, D. Paul, T. M. Buzug, D. L. G. Hill, and D. J. Hawkes, "Voxel-based 2-D/3-D registration of fluoroscopy images and ct scans for image-guided surgery," IEEE Trans. Inform. Technol. Biomed., vol. 1, pp. 284-293, Dec. 1997.

[9] S. Warfield, A. Robatino, J. Dengler, F. Jolesz, and R. Kikinis, "Nonlinear registration and template-driven segmentation," in Brain Warping, A. W. Toga, Ed. New York: Academic, 1999, pp. 67-84.
[10] R. Szeliski and J. Coughlan, "Spline-based image registration," Int. J. Comput. Vis., vol. 22, pp. 199-218, 1997.

[11] O. Faugeras and R. Keriven, "Variational principles, surface evolution, PDE's, level set methods, and the stereo problem," IEEE Trans. Image Processing, vol. 7, pp. 336-344, Mar. 1998.

[12] B. McGregor, "Automatic registration of images of pigmented skin lesions," Pattern Recognit., vol. 31, no. 6, pp. 805-817, 1998.

[13] M. Gabrani and O. J. Tretiak, "Surface-based matching using elastic transformations," Pattern Recognit., vol. 32, pp. 87-97, 1999.

[14] U. Kjems, S. C. Strother, J. Anderson, I. Law, and L. K. Hansen, "Enhancing the multivariate signal of ${ }^{15} \mathrm{O}$ water PET studies with a new nonlinear neuroanatomical registration algorithm," IEEE Trans. Med. Imag., vol. 18, pp. 306-319, Apr. 1999.

[15] C. Nikou, F. Heitz, and J.-P. Armspach, "Robust voxel similarity metrics for the registration of dissimilar single and multimodal images," Pattern Recognit., vol. 32, pp. 1351-1368, 1999.

[16] F. Maes, A. Collignong, D. Vandermeulen, G. Marchal, and P. Suetens, "Multimodality image registration by maximization of mutual information," IEEE Trans. Med. Imag., vol. 16, no. 2, pp. 187-198, Apr. 1997.

[17] P. Thévenaz and M. Unser, "Optimization of mutual information for multiresolution image registration," IEEE Trans. Image Processing, vol. 9, pp. 2083-2099, Dec. 2000.

[18] J. Ashburner and K. J. Friston, "Nonlinear spatial normalization using basis functions," in Brain Warping, A. W. Toga, Ed. New York: Academic, 1999, pp. 254-266.

[19] J. Gee, "On matching brain volumes," Pattern Recognit., no. 32, pp. 99-111, 1999

[20] J. Martin, A. Pentland, S. Sclaroff, and R. Kikinis, "Characterization of neuropathological shape deformations," IEEE Trans. Pattern Anal. Machine Intell., vol. 2, Feb. 1998.

[21] K. V. Asari, K. Sanjiv, and D. Radhakrishnan, "A new approach for nonlinear distortion correction in endoscopic images based on least squares estimation," IEEE Trans. Med. Imag., vol. 18, p. 345, Apr. 1999.

[22] J. Kybic, P. Thévenaz, A. Nirkko, and M. Unser, "Unwarping of unidirectionally distorted EPI images," IEEE Trans. Med. Imag., vol. 19, pp. 80-93, Feb. 2000

[23] F. Yeung, F. Levinson, D. Fu, and K. J. Parker, "Feature-adaptive motion tracking of ultrasound image sequences using a deformable mesh," IEEE Trans. Med. Imag., vol. 17, pp. 945-956, Dec. 1998.

[24] D. Suter and F. Chen, "Left ventricular motion reconstruction based on elastic vector splines," IEEE Trans. Med. Imag., vol. 19, pp. 295-305, Apr. 2000.

[25] P. Rösch, J. Weese, T. Netsch, M. Quist, G. Penney, and D. Hill, "Robust 3D deformation field estimation by template propagation," in Proc. MICCAI, 2000, pp. 521-530.

[26] P. A. van den Elsen, E.-J. D. Pol, and M. A. Viergever, "Medical image matching-A review with classification," IEEE Eng. Med. Biol., pp. 26-39, Mar. 1993.

[27] J. Maintz and M. A. Viergever, "A survey of medical image registration," Med. Imag. Anal., vol. 2, no. 1, pp. 1-36, 1998.

[28] H. Lester and S. R. Arridge, "A survey of hierarchical nonlinear medical image registration," Pattern Recognit., vol. 32, no. 1, pp. 129-149, Jan. 1999.

[29] G. Christensen, S. Joshi, and M. Miller, "Volumetric transformation of brain anatomy," IEEE Trans. Med. Imag., vol. 16, pp. 864-877, Dec. 1997.

[30] M. Miller, S. Joshi, and G. Christensen, "Large deformation fluid diffeomorphisms for landmark and image matching," in Brain Warping, A. Toga, Ed. New York: Academic, 1999, pp. 115-132.

[31] G. Christensen and H. Johnson, "Consistent image registration," IEEE Trans. Med. Imag., vol. 20, pp. 568-582, July 2001.

[32] G. Hermosillo. (2002) Variational methods for multimodal image matching, Ph.D. dissertation. INRIA. [Online]. Available: ftp://ftp-sop. inria.fr/robotvis/html/Papers/hermosillo:02.ps.gz

[33] O. Musse, F. Heitz, and J.-P. Armspach, "Topology preserving deformable image matching using constrained hierarchical parametric models," IEEE Trans. Med. Imag., vol. 10, pp. 1081-1093, July 2001.

[34] F. Heitz, P. Perez, and P. Bouthemy, "Multiscale minimization of global energy functions in some visual recovery problems," Comput. Vis. Graph. Image Process., vol. 59, no. 1, pp. 125-134, 1994.

[35] H. Yoshida, "Removal of normal anatomic structures in radiographs using wavelet-based non-linear variational method for image matching," Wavelet Applicat. Signal Image Process., vol. 3458, pp. 174-181, 1998.

[36] Y. Wu, T. Kanade, C. Li, and J. Cohn, "Image registration using waveletbased motion model," in Int. J. Comput. Vis., J. Le Moigne, Ed., 2000, vol. 38, pp. 129-152. 
[37] J. Kybic and M. Unser, "Multidimensional elastic registration of images using splines," in Proc. ICIP Vancouver, BC, Canada, 2000, vol. II, pp. $455-458$

[38] J. Kybic. Biomedical image processing by elastic warping, Ph.D. dissertation. Ecole Polytechnique Fédérale de Lausanne, Lausanne, Switzerland. [Online]. Available: http://cmp.felk.cvut.cz/ kybic/research.html.

[39] K. Rohr, H. S. Stiehl, R. Sprengel, W. Beil, T. M. Buzug, J. Weese, and M. H. Kuhn, "Point-based elastic registration of medical image data using approximating thin-plate splines," in Visualization in Biomedical Computing, K. H. Höhne and R. Kikinis, Eds. Berlin, Germany: Springer-Verlag, 1996, pp. 297-306.

[40] F. L. Bookstein, "Principal warps: Thin-plate splines and the decomposition of deformations," IEEE Trans. Pattern Anal. Machine Intell., vol. 6, pp. 567-585, June 1989.

[41] J. P. W. Pluim, J. B. A. Maintz, and M. A. Viergever, "Image registration by maximization of combined mutual information and gradient information," IEEE Trans. Med. Imag., vol. 19, pp. 809-814, Aug. 2000.

[42] M. Unser, "Splines: A perfect fit for signal and image processing," IEEE Signal Processing Mag., vol. 16, pp. 22-38, Nov. 1999.

[43] W. H. Press, S. A. Teukolsky, W. T. Vetterling, and B. P. Flannery, Numerical Recipes in $C$, 2 nd ed. Cambridge, U.K.: Cambridge Univ. Press, 1992.

[44] J. Kybic, P. Thévenaz, and M. Unser, "Compensation of unidirectional geometric distortion in EPI using spline warping," in Proc. IEEE Int. Conf. Image Processing, Kobe, Japan, 1999, pp. 168-172.

[45] N. Sicotte, R. Woods, and J. Mazziotta, "Automated image registration using a 105 parameter nonlinear model," in Neuroimage, Proc. 2nd Int. Conf. Functional Mapping of the Human Brain, vol. 3, June 1996, pp. $153-165$

[46] S. Kiebel, J. Ashburner, J. Poline, and K. Friston, "MRI and PET coregistration-A cross validation of statistical parametric mapping and automated image registration," Neuroimage, no. 5, 1997.

[47] M. Fornefett, K. Rohr, and H. S. Stiehl, "Elastic registration of medical images using radial basis functions with compact support," in Proc. Computer Vision and Pattern Recognition (CVPR'99), Fort Collins, CO, 1999, pp. 402-407.

[48] Y. Amit, "A nonlinear variational problem for image matching," SIAM J. Sci. Comput., vol. 15, no. 1, pp. 207-224, 1994.

[49] Y.-T. Wu, "Image registration using wavelet-based motion model and its applications," Ph.D. dissertation, Univ. Pittsburgh, Saint Louis, MO, 1997.

[50] D. Sutter and F. Chen, "Using a fast multipole method to accelerate the evaluation of splines," IEEE Comput. Sci. Eng., vol. 3, pp. 24-31, July 1998.

[51] R. K. Beatson and G. Newsam, "Fast evaluation of radial basis functions: I," Comput. Math. Applicat., vol. 24, no. 12, pp. 7-19, 1992.

[52] R. K. Beatson and M. J. D. Powell, "An iterative method for thin-plate spline interpolation that employs approximations to the lagrange functions," in Numerical Analysis 1993, D. F. Griffiths and G. A. Watson, Eds. Essex, U.K.: Longman, 1994, pp. 17-39.

[53] C. R. Anderson, "An implementation of the fast multipole method without multipoles," SIAM J. Sci. Comput., vol. 13, no. 4, pp. 923-947, July 1992.

[54] M. Unser, A. Aldroubi, and M. Eden, "A family of polynomial spline wavelet transforms," Signal Process., vol. 30, no. 2, pp. 141-162, Jan. 1993.

[55] I. Schoenberg, "Spline functions and the problem of graduation," Proc. Nat. Acad. Sci., vol. 52, pp. 947-950, 1964.

[56] J. H. Ahlberg, E. N. Nilson, and J. L. Walsh, The Theory of Splines and Their Applications. New York: Academic, 1967.

[57] P. Thévenaz, U. E. Ruttimann, and M. Unser, "A pyramid approach to subpixel registration based on intensity," IEEE Trans. Image Processing, vol. 7, pp. 1-15, Jan. 1998
[58] P. Cachier et al., "Multisubject nonrigid registration of brain MRI using intensity and geometric features," in Proc. MICCAI, Utrecht, The Netherlands, 2001, pp. 734-742.

[59] P. Hellier and C. Barillot, "Cooperation between local and global approaches to register brain images," in Proc. IPMI, 2001, pp. 315-328.

[60] M. Unser, A. Aldroubi, and M. Eden, "The $L 2$ polynomial spline pyramid," IEEE Trans. Pattern Anal. Machine Intell., vol. 15, Apr. 1993.

[61] M. Unser and T. Blu, "Fractional splines and wavelets," SIAM Rev., vol. 42 , no. 1 , pp. 43-67, 2000.

[62] P. Flandrin, "Wavelet analysis and synthesis of fractional Brownian motion," IEEE Trans. Inform. Theory, vol. 38, no. 2, pp. 910-917, Mar. 1992.

[63] S. Mallat, A Wavelet Tour of Signal Processing. San Diego, CA: Academic, 1998.

[64] I. Kanno and N. Lassen, "Two methods for calculating regional cerebral blood flow from emission computed tomography of inert gas concentrations," J. Comput. Assist. Tomogr., vol. 1, no. 3, pp. 71-76, 1979.

[65] M. J. Ledesmay-Carbayo, J. Kybic, M. Desco, A. Santos, and M. Unser, "Cardiac motion analysis from ultrasound sequences using nonrigid registration," in Proc. MICCAI, W. J. Niessen and M. A. Viergever, Eds., Utrecht, The Netherlands, Oct. 2001, pp. 889-896.

[66] J.-P. Vallée et al., "MRI quantitative myocardial perfusion with compartmental analysis: A rest and stress study," Magn. Reson. Med., vol. 38, pp. 981-989, 1997.

[67] _ "Quantification of myocardial perfusion with FAST sequence and Gd bolus in patients with normal cardiac function," J. Magn. Reson. Imag., vol. 9, pp. 197-203, 1999.

Jan Kybic (M'00) was born in Prague, Czech Republic, in 1974. He received the Bc. (B.Sc.) and Ing. (M.Sc.) degrees with honors from the Czech Technical University, Prague, in 1996 and 1998, respectively. In 2001, he received the Ph.D. degree in biomedical image processing from Ecole Polytechnique Federale de Lausanne (EPFL), Switzerland, for his dissertation on elastic image registration using parametric deformation models.

Between October 2002 and February 2003, he held a post-doc research position in INRIA, Sophia-Antipolis, France. Currently, he is with Center for Machine Perception, Czech Technical University. His research interests include signal and image processing in general, image registration, splines and wavelets, speech processing and enhancement, computer vision, numerical methods, algorithm theory, and control theory.

Michael Unser (M'89-SM'94-F'99) received the M.S. (summa cum laude) and $\mathrm{Ph} . \mathrm{D}$. degrees in electrical engineering in 1981 and 1984, respectively, from the Swiss Federal Institute of Technology (EPFL), Lausanne, Switzerland.

From 1985 to 1997, he was with the Biomedical Engineering and Instrumentation Program, National Institutes of Health, Bethesda, MD. He is now Professor and Head of the Biomedical Imaging Group at EPFL. His main research area is biomedical image processing. He has a strong interest in sampling theories, multiresolution algorithms, wavelets, and the use of splines for image processing. He is the author of 90 published journal papers in these areas. He was on the editorial board of Signal Processing.

Dr. Unser is an Associate Editor for the IEEE TRAnsactions ON MEDICAL IMAGING. He has been on the editorial boards the IEEE TRANSACTIONS ON IMAge PROCESSING from 1992 to 1995 and the IEEE Signal PROCESSING LETTERS from 1994 to 1998. He serves as regular chair for the SPIE Conference on Wavelets, which has been held annually since 1993 . He received the 1995 Best Paper Award and the 2000 Magazine Award from the IEEE Signal Processing Society. 